\title{
Artikel
}

Christine Göttler*

\section{Die Fruchtbarkeit der Bilder: Kopieren nach Dürer um 1600}

Eine erste Version dieses Beitrags wurde an der im Mai 2013 von Ulrich Heinen und Johann Anselm Steiger an der Universität Hamburg organisierten Konferenz "Das Gebet in den Konfessionen und Medien der Frühen Neuzeit" vorgetragen. Mein Dank geht an die Teilnehmenden sowie besonders an Ulrich Heinen für zahlreiche Anregungen zur Weiterentwicklung des Themas.

(ङ) Br-NC-ND (C) 2019 Christine Göttler, licensee De Gruyter Open. This work is licensed under the Creative Commons Attribution-NonCommercial-NoDerivs 3.0 License. 
Abstract: In der künstlerischen Praxis der Frühen Neuzeit kam dem Nachahmen, Kopieren, Reproduzieren und ,Fälschen' anderer Werke eine zentrale Bedeutung zu. Die kunstgeschichtliche Forschung hat sich in den vergangenen Jahren vermehrt mit solchen Übersetzungs- und Transformationsprozessen beschäftigt, welche die Unterscheidung von Original und Imitat problematisieren. Dieser Beitrag befasst sich am Beispiel einer vielfach kopierten Zeichnung Albrecht Dürers mit unterschiedlichen Verfahren der Wiederholung, Aneignung, Verwandlung und Reproduktion an der Schwelle vom 16. zum 17. Jahrhundert, wie sie in den entstehenden fürstlichen Sammlungen praktiziert wurden. Im Vordergrund stehen intermediale und interpikturale Strategien der Aneignung, die sich auch an der künstlerischen Eigenart und Technik orientierten, sowie Fragen frühneuzeitlicher musealer Aufbewahrung und Präsentation.

Keywords: Kopie, „principael”, Kunstkammer, Werkstatt, museale Präsentation, Jan Brueghel der Ältere, Michiel Coxcie, Albrecht Dürer, Jan van Eyck, Lucas de Heere, Leonardus Lessius, Karel van Mander, Jacob Matham, Aegidius Sadeler, Rudolf II.

*Prof. em. Dr. Christine Göttler: Institut für Kunstgeschichte, Abteilung für Kunstgeschichte der Neuzeit, Universität Bern, Mittelstrasse 43, CH-3012 Bern, email: christine.goettler@ikg.unibe.ch

An der Wende vom 16. zum 17. Jahrhundert entwickelte sich ein neues Gespür für die Fragilität der materiellen Kultur; es entstand eine neue Wertschätzung gegenüber Bildern und Objekten, welche die umfassenden Zerstörungen des reformatorischen Ikonoklasmus (und der fortdauernden Kriege) überlebt hatten. Diese äußerte sich sowohl im Ort, der alten religiösen Bildern in den neu entstehenden Sammlungen zugewiesen wurde, als auch in den zahlreichen Kopien, Übertragungen und Überarbeitungen dieser Werke. Die neuen, nachkommenden Bilder orientierten sich einerseits an der kompositorischen Struktur und ikonographischen Formel, andererseits an der künstlerischen Eigenart und Technik der alten Bilder, die manchmal auch in ein anderes Medium und Material übersetzt wurden. ${ }^{1}$ Solche medialen und materiellen Transformationsprozesse stehen im Zentrum dieses Beitrags, der am Beispiel der Rezeption von Albrecht Dürers Zeichnung des Großen Kalvarienbergs das ,Fortleben' von religiösen Bildern, die mit affektiven Werten verknüpft waren, in der einzelnen oder seriellen Kopie untersucht. Die Verwendung

1 Vgl. Bushart 2018. anderer Medien, Materialien und Techniken entspricht, so soll hier als These formuliert werden, einer Reformierung, Aktualisierung und Intensivierung künstlerischer Prozesse. Im Kontext eines neu entstehenden Kunstmarkts und einer sich dynamisierenden Sammlungskultur werden die Wirkungen und Werte künstlerischer Medien, Materialien und Techniken neu verhandelt. Weiter liegt der Fokus auf einer sehr spezifischen Form der Kopie, die sich explizit durch Inschriften und Signaturen als solche zu erkennen gibt und die dem Kopisten einen Teil der Autorschaft zuerkennt. Diese Form der Kopie unterscheidet sich einerseits von der Fälschung, einer in betrügerischer Absicht verfertigten Kopie, andererseits von jenen zahllosen Bildern, die in Inventaren des 17. Jahrhunderts als Kopien aufgelistet und dem originalen Künstler oder dessen Werkstatt zugeschrieben werden. ${ }^{2}$

2 Das vom 10. April 1614 datierte Inventar des Antwerpener Kunstsammlers Philips van Valckenisse listet unter der Überschrift "copiën” eine ganze Reihe von als Kopien gehandelten Gemälden. Außerdem werden sechs Kopien nach Werken von Pieter Bruegel dem Älteren genannt, die seinem Sohn, Pieter Brueghel dem Jüngeren zugeschrieben werden, der sich auf die Reproduktion der Werke sei- 
Die relativ großformatige, 1505 datierte Federund Pinselzeichnung des Großen Kalvarienbergs auf grün grundiertem Papier zitiert die für das späte 15. Jahrhundert charakteristische Ikonographie des ,vielfigurigen' oder, volkreichen' Kalvarienbergs, der Dürer jedoch eine neue Lebendigkeit und Dramatik verleiht (Abb. 1). ${ }^{3}$ Dargestellt ist die Passion Jesu auf Golgatha in verschiedenen Episoden auf einer bühnenähnlichen, nach hinten ansteigenden Ebene. Im Bildvordergrund sind die Vorbereitungen zur Kreuzannagelung zu sehen jene Episoden, die in besonderer Weise bei den Betrachtern Mitleid und die Vorstellung der eigenen Schuld hervorrufen sollten und die auch in den gängigen Meditationstraktaten etwa von Ludolf von Sachsen und Pseudo-Bonaventura behandelt sind. ${ }^{4}$ Die Komposition ist auf der kleinen Tafel unterhalb der beiden miteinander ringenden Soldaten mit dem Monogramm Dürers und der Jahreszahl 1505 versehen (Abb. 2). Wegen ihres ungewöhnlichen narrativen Reichtums wurde die Zeichnung in der älteren Forschungsliteratur bisweilen aus Dürers Fuvre ausgeschlossen, gilt jedoch heute bei den meisten Experten als eigenhändig. ${ }^{5}$ Im 16. und 17. Jahrhundert gehörte Dürers Großer Kalvarienberg zu den am meisten bewunderten und am häufigsten zitierten Werken des Künstlers. Da die ersten Bildzitate in den frühen 1520 er Jahren in Leiden auftraten, ist schon vermutet worden, dass sich die Zeichnung unter jenen Werken befand, die Dürer auf seiner Reise in die Niederlande 1520/21 dem Zeichner und Stecher Lucas van Leyden zeigte, mit dem er

nes Vaters spezialisierte. Duverger 1984, S. 308-311. Auch das Inventar der Kunstkammer Kaiser Rudolfs II. unterscheidet zwischen Originalen und Kopien: Bauer / Haupt 1976. Siehe unten, Anm. 40.

3 Zum vielfigurigen Kalvarienberg: Roth 1958; Suckale 2009, Bd. 1, S. 81-101. Zur fortdauernden Verwendung der Ikonographie des, volkreichen' Kalvarienbergs in der Zeit Dürers: Silver 2008, S. 211. Vgl. auch Hess / Eser 2012, S. 405, Kat. 96.

4 Marrow 1979, S. 164-170.

5 Silver 2008, S. 209-223; Strauss 1974, Bd. 2, S. 846; Winkler 1937, Bd. 2, S. 30, 40-43, Nr. 317. Winkler vermutete, dass eine genauere Untersuchung der Zeichnung "das hohe Ansehen bestätigt, in dem die Komposition ehedem stand [und] dem Werk einen Ehrenplatz in Dürers Werk verschaffen" wird. Er berichtigte dadurch eine frühere Stellungnahme, in der er die Zeichnung nicht als eigenhändig gelten ließ: Winkler 1929, S. 123-166, hier S. 164-65. mehrere Werke austauschte und sich über Zeichnungstechniken unterhielt. ${ }^{6}$

Es geht hier jedoch um jene Kette von Übertragungen und Transformationen, die mit der Aufnahme des Werkes in die kaiserlichen Sammlungen in Prag rund hundert Jahre nach seiner Entstehung einsetzte. Wie auch Karel van Mander im Schilder-Boeck von 1604 vermerkte, wurden Zeichnungen von Dürers "kunstreicher Hand" in jenen Jahren intensiv gesammelt, ${ }^{7}$ wobei Kaiser Rudolf II., der "gegenwärtig größte Verehrer der Malkunst der Welt", als der wohl leidenschaftlichste und mit Sicherheit mächtigste Bewunderer von Dürers Kunst galt. ${ }^{8}$ Dürer begann kurz nach 1500 farbig grundiertes Papier zu verwenden. ${ }^{9}$ Eine vergleichbare Technik zur Erzeugung von Reliefwirkung durch Hell-Dunkel-Kontraste wurde von Dürer auch in der sogenannten Grünen Passion von 1504 angewendet, die Joachim von Sandrart in der Teutsche[n] Akademie von 1675 als die "beste aller seiner Passionen" bezeichnete und die ebenfalls in den Besitz Rudolfs II. gelangte. ${ }^{10}$ Dürer bezog sich durch die Kombination von zeichnerischen und malerischen Verfahren auch auf ein frühes Stadium der Malerei: der einfarbigen oder monochromen Malerei, wie sie Plinius und Quintilian mit unterschiedlichen Akzenten für die Antike diskutiert haben. ${ }^{11} \mathrm{Zu}$ Beginn des 16. Jahrhunderts verband sich die Forderung, auf den Luxus vieler Farben zu verzichten, mit der Forderung nach religiöser Reform. ${ }^{12}$ Erasmus von Rotterdam setzte in einer oft zitierten Stelle Dürers "schwarze Striche” mit den "monochromata" (dem Einfarbigen) der antiken Maler

6 Leidener Schule, Kalvarienberg, um 1520. Öl auf Eichenholz, 172,5 x 119 cm. Amsterdam, Rijksmuseum, Inv.-Nr. SK-A-4921. Filedt Kok 1996; Van Os 1996; Van Os 2001; Silver 2008, S. 216. Nach Silver könnten die Figuren im linken Vordergrund von Lucas van Leyden gemalt worden sein. Vgl. auch Lossky 1959.

7 Van Mander 1604, Fol. 208v: "Veel teyckeninghen zijn oock by verscheyden Const-beminders te sien."

8 DaCosta Kaufmann 1985, S. 22-39.

9 White 1971, S. 19.

10 Sandrart 1675, S. 224 (2. Teil, 3. Buch): „ein Buch in Quart, darinn der ganze Paßion / auf grün Papier mit der Feder / gezeichnet / und Bleyweiß gehöcht / welches von allen seinen Paßionen für die bäste zu halten." Sandrart sah die Zeichungen in der "Kunst-Cammer zu Wien”.

11 Plinius 1997, S. 22-23 (35 V, 16); Quintilianus 1995, Bd. 2, S. 754-755 (XII 10, 3).

12 Göttler 1990, S. 287-291. 


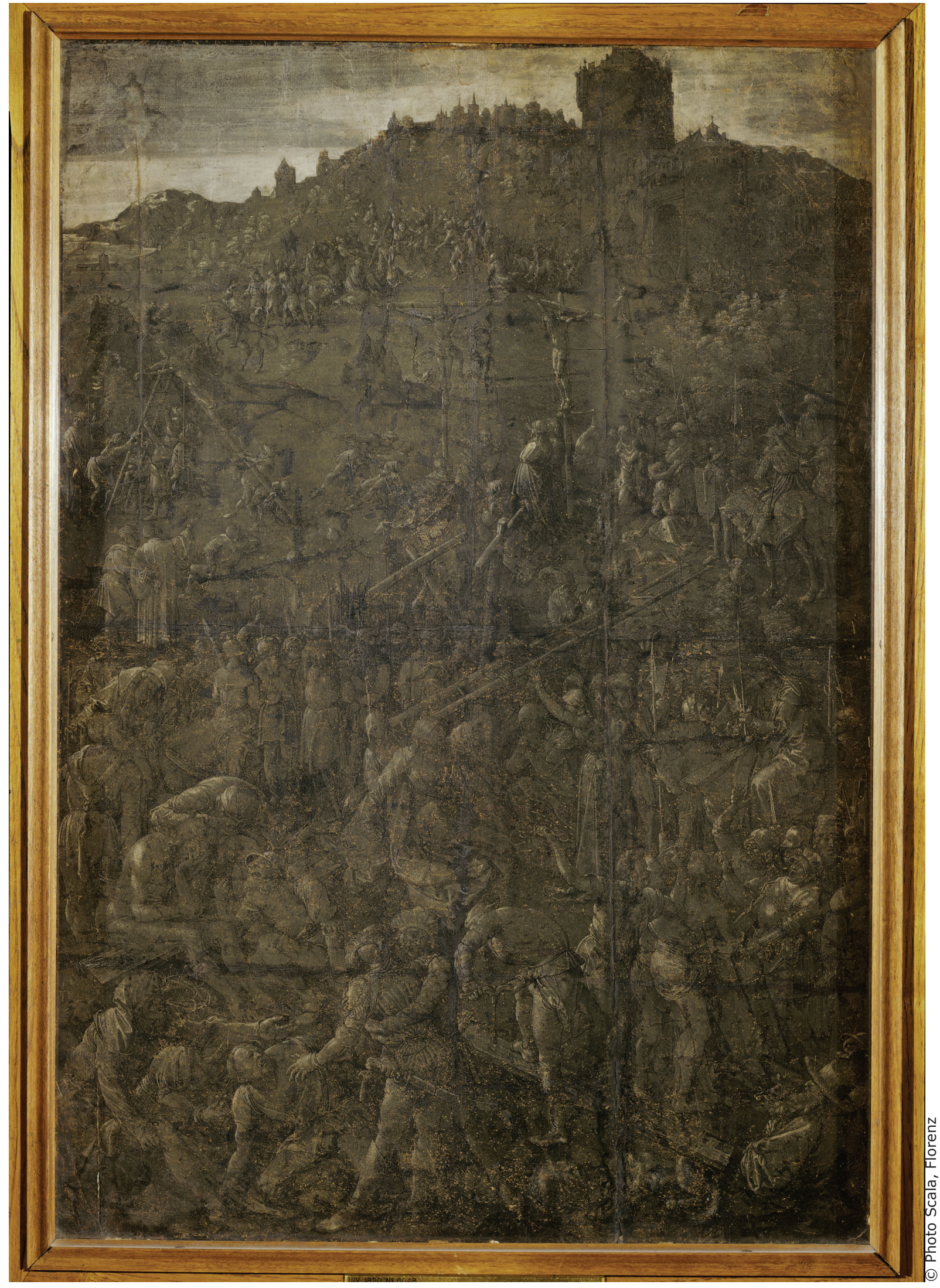

Abb. 1. Albrecht Dürer, Der Große Kalvarienberg, 1505. Feder- und Pinselzeichnung auf grün grundiertem Papier, weiß gehöht, schwarz laviert, aufgezogen auf Holz, 58 x $40 \mathrm{~cm}$. Florenz, Galleria degli Uffizi, Gabinetto dei Disegni e delle Stampe, Inv.-Nr. 1890 n. 8406. 


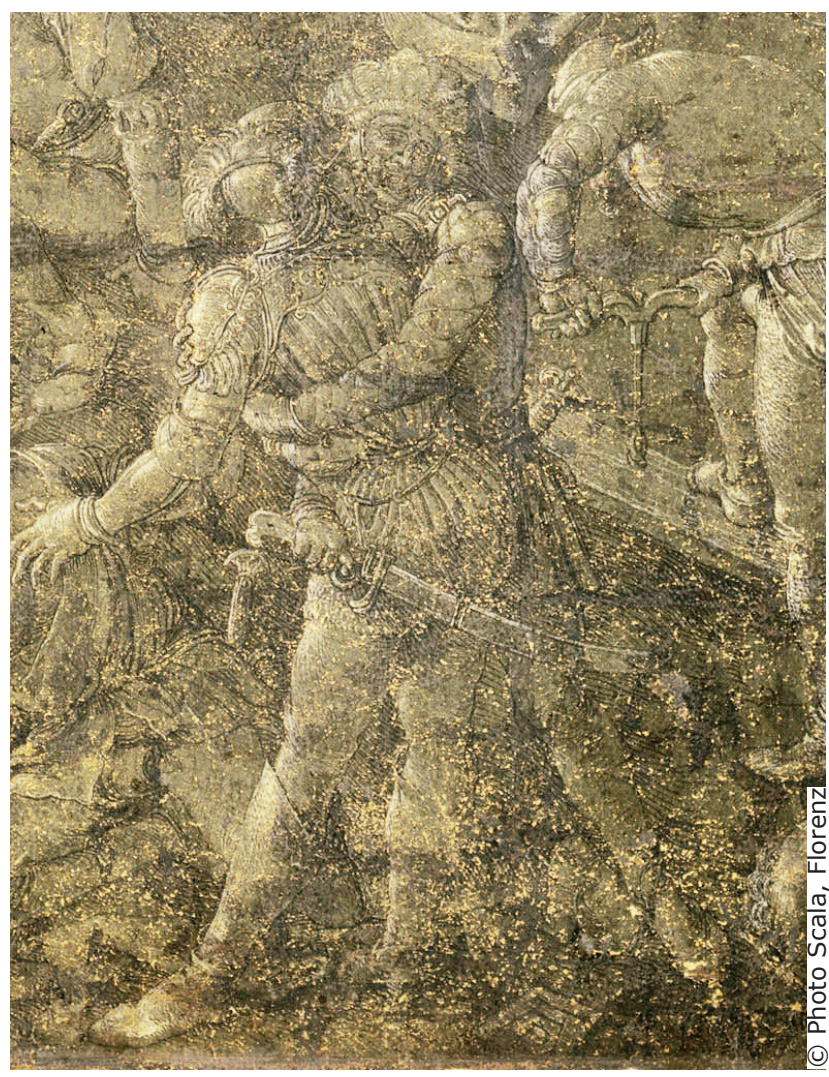

Abb. 2. Detail von Abb. 1: Monogramm Dürers (zur besseren Lesbarkeit digital aufgehellt).

gleich. War Apelles auf Farben angewiesen, so vermochte Dürer "mit schwarzen Strichen" alles auszudrücken, auch dasjenige, das sich nicht malen lässt (wie "Donnerschläge, Wetterleuchten, Nebelwände"). ${ }^{13}$

Am Hof Rudolfs II. in Prag entstand nun auch das vom Antwerpener Maler Jan Brueghel dem Älteren entworfene Gemälde (Abb. 3, 4, 5). Offensichtlich sollte es in direkter Verbindung mit Dürers Zeichnung des Großen Kalvarienbergs gelesen werden, die wohl schon damals recht schadhaft war. Dürers Zeichnung und Brueghels gemalte Kopie wurden wohl schon in Prag zu einem Diptychon zusammengefügt; dieses gelangte im frühen 17. Jahrhundert in die mediceischen Sammlungen in Florenz, wo es

13 Erasmus Roterodamus 1528, S. 69: „At Apelles coloribus licet paucioribus minusque ambitiosis, tamen coloribus adiuvabatur. Durerus quanquam et alias admirandus, in monochromatis, hoc est, nigris lineis, quid non exprimit? umbras, lumen, splendorem, eminentias, depressiones [...]. Quin ille pingit et quae pingi non possunt, ignem, radios, tonitrua, fulgetra, fulgura, vel nebulas [...]." Vgl. Panofsky 1951. sich noch heute befindet. Dürers Komposition ist darüber hinaus durch zwei weitere Werke des frühen 17. Jahrhunderts überliefert, die sich wie Brueghels Gemälde in Bezug auf Größe und Detail an Dürers Entwurf orientierten und auf diesen auch ausdrücklich verweisen: Es handelt sich dabei um einen 1615 datierten Kupferstich Jacob Mathams, des Adoptivsohns von Hendrick Goltzius, der sich gerade in seinen graphischen Werken mehrfach von Dürer hatte anregen lassen (Abb. 6, 7); und ein Holzrelief eines unbekannten Bildschnitzers (Abb. 9). Im Folgenden sollen drei Aspekte hervorgehoben werden, die in der bisherigen Literatur zur Rezeption von Dürers Zeichnung wenig beachtet worden sind: die Eigenheiten und Möglichkeiten der spezifischen künstlerischen Verfahren, die einerseits Dürers Zeichnung neu zur Wirkung bringen beziehungsweise sie neu lesbar machen und die andererseits auf die Kunst oder Kunstfertigkeit der Nachahmer oder vielmehr der ,zweiten' Erfinder oder Urheber aufmerksam machen; die Art und Weise, wie mittels Monogrammen und Signaturen mehrfache oder geteilte Urheberschaft inszeniert wird; und die enge Verflechtung von religiöser und künstlerischer Imitation, oder von meditativen und künstlerischen Aneignungsprozessen: in anderen Worten, die Interdependenz der künstlerischen Aneignung von Dürers Meditationsbild der Passion und der betrachtenden Aneignung und Wiederholung der Passion in der Meditation.

Wie aber lässt sich das Verhältnis zwischen Dürers Zeichnung und deren Übertragungen in die Medien der Ölmalerei, des Kupferstichs und des Holzreliefs begrifflich fassen? Hält man sich die Schadhaftigkeit und die damit verbundene Unlesbarkeit der Zeichnung vor Augen, so ermöglichten die Übertragungen in haltbarere Medien im Sinne von Walter Benjamins Abhandlung zur Aufgabe des Übersetzers das „Fortleben des Originals".14 Die Kopien des rund hundert Jahre zuvor geschaffenen Werkes erkundeten dessen mimetische Möglichkeiten in den eigenen künstlerischen Medien und öffneten den Blick für ein historisches Verständnis von Kunst, das nicht linear sondern iterativ definiert war und mit Verweisen, Rückbezügen und Neubewertungen arbeitete. So verstand auch Van Mander im Schil-

$14 \mathrm{Vgl}$. den Beitrag von Ludwig Jäger in diesem Heft. 


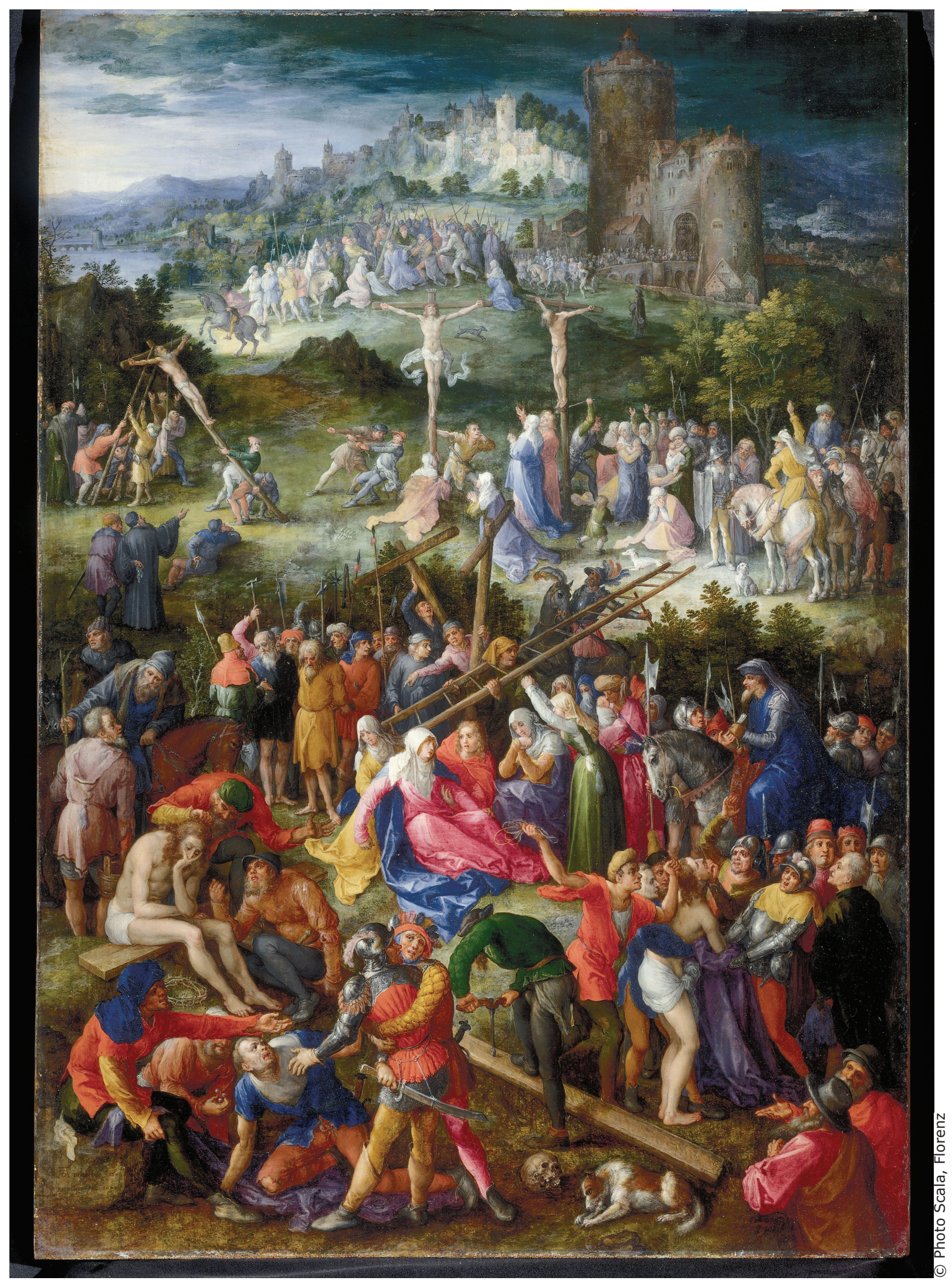

Abb. 3. Jan Brueghel der Ältere, Der Große Kalvarienberg (nach Albrecht Dürer), 1604. Öl auf Eichenholz, 62 x 42 cm. Florenz, Galleria degli Uffizi, Inv.-Nr. 1890 n. 1083. 


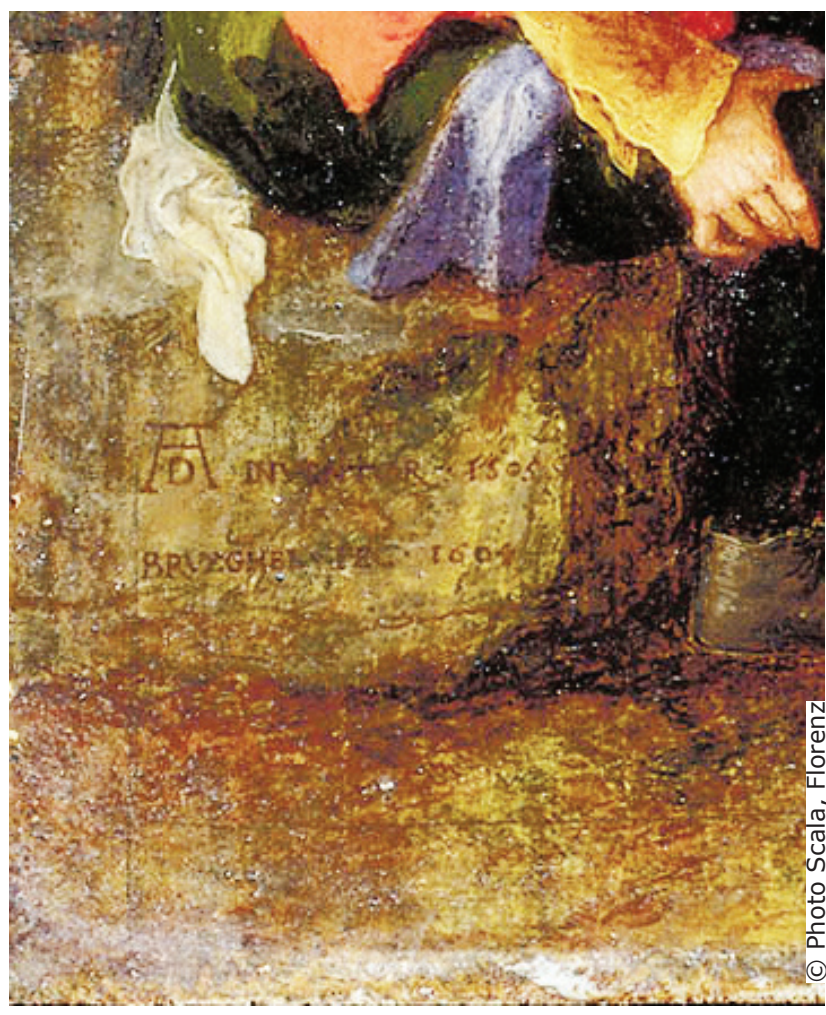

Abb. 4. Detail von Abb. 3. Die Signatur Jan Brueghels (zur besseren Lesbarkeit digital aufgehellt).

der-Boeck die ,Erfindung' der Ölmalerei durch Jan van Eyck als das zentrale Gründungsereignis der niederländischen Malerei, auf das sich alle nachfolgenden Maler in ihren Werken wiederholend beziehen konnten: „Es war da ein neues Geschlecht und eine neue Art von Werken geboren worden." ${ }^{15}$ Van Mander schrieb die Geschichte der niederländischen Kunst weniger als eine Fortschrittsgeschichte denn vielmehr als eine Weiterentwicklung einer entscheidenden künstlerischen Erfindung in unterschiedliche Richtungen. Für ihn war die Ölmalerei der "Gipfel der Kunst und das allerbeste Mittel, um die Natur in allen Teilen auf das ähnlichste nachzubilden". ${ }^{16}$

15 Van Mander 1604, Fol. 199v: "want hier is gheboren een nieuw gheslacht / en gedaente van wercken". Vgl. Göttler 2017.

16 Van Mander 1604, Fol. 294r: „als wesende het opperste der Const / en den alder bequaemsten middel / om de Natuere in allen deelen met uytbeeldinghe ten alder ghelijcksten nae te comen".

\section{Der Wert der Kopie}

Alexander Nagel und Christopher Wood haben in ihrem 2010 veröffentlichten Buch Anachronic Renaissance Kopien von Kultbildern von jenen seit dem späten 15. Jahrhundert nachweisbaren exakten Kopien alter Gemälde unterschieden, die von untereinander rivalisierenden Kunstliebhabern in Auftrag gegeben wurden. ${ }^{17}$ Im Unterschied zur Replik eines Kultbildes, die lediglich die wesentlichen ikonographischen Merkmale des Originals aufweisen musste, um dessen Wirkung in anderen Kontexten und Räumen zu entfalten, erforderte ein Sammlerbild eine weitaus größere Angleichung an das Original, die auch motivische und stilistische Besonderheiten umfasste. Eine exakte Kopie, welche die Erscheinung eines Originals bis in das kleinste Detail nachzuahmen versucht, um so selbst als Original wahrgenommen zu werden, gerät allerdings in die Nähe einer Fälschung; sie täuscht eine falsche Entstehungsgeschichte vor. ${ }^{18}$

In unserem Zusammenhang von besonderem Interesse ist Michiel Coxcies in den originalen Maßen und im selben Medium geschaffene Kopie des Genter Altars der Brüder Van Eyck, des zentralen Referenzwerkes für die niederländische Malerei. Die außerordentlich hohe Summe von schätzungsweise 4.000 Gulden, die Coxcie angeblich für das von Philipp II. beauftragte Werk erhielt, dokumentiert die Wertschätzung seiner Arbeit, die rund zwei Jahre (von Anfang Januar 1556 bis Ende Oktober 1558) in Anspruch nahm und in der Vijd-Kapelle selbst, also vor dem Original, ausgeführt wurde. ${ }^{19}$ Stephan Kemperdick hat die staunenerregende Genauigkeit hervorgehoben, mit der Coxcie „jede Feinheit, jedes Glanzlicht" des originalen Werkes wiederholte und zudem vermutet, dass Pausen verwendet wurden. ${ }^{20}$ Van Mander beschreibt die Anstrengungen, die man aufbot, um über Tizian in Venedig das

17 Nagel / Wood 2010, S. 275-287.

18 Nagel / Wood 2010, S. 281. Eine vergleichbare Argumentation findet sich auch bei Ariane Mensger (Mensger 2010), welche die "exakte Kopie" als ein frühneuzeitliches Phänomen versteht, die einerseits die Rezeption alter Werke lenkte und andererseits ein neues Künstlerbild in eine vergangene Zeit zurückprojiziert.

19 Suykerbuyk 2017; Suykerbuyk 2013, S. 5-10; Kemperdick 2014, S. 42-50; Putzger 2019; Putzger 2018, S. 109-114. 20 Kemperdick 2014, S. 47; vgl. auch Dubois 2017. 


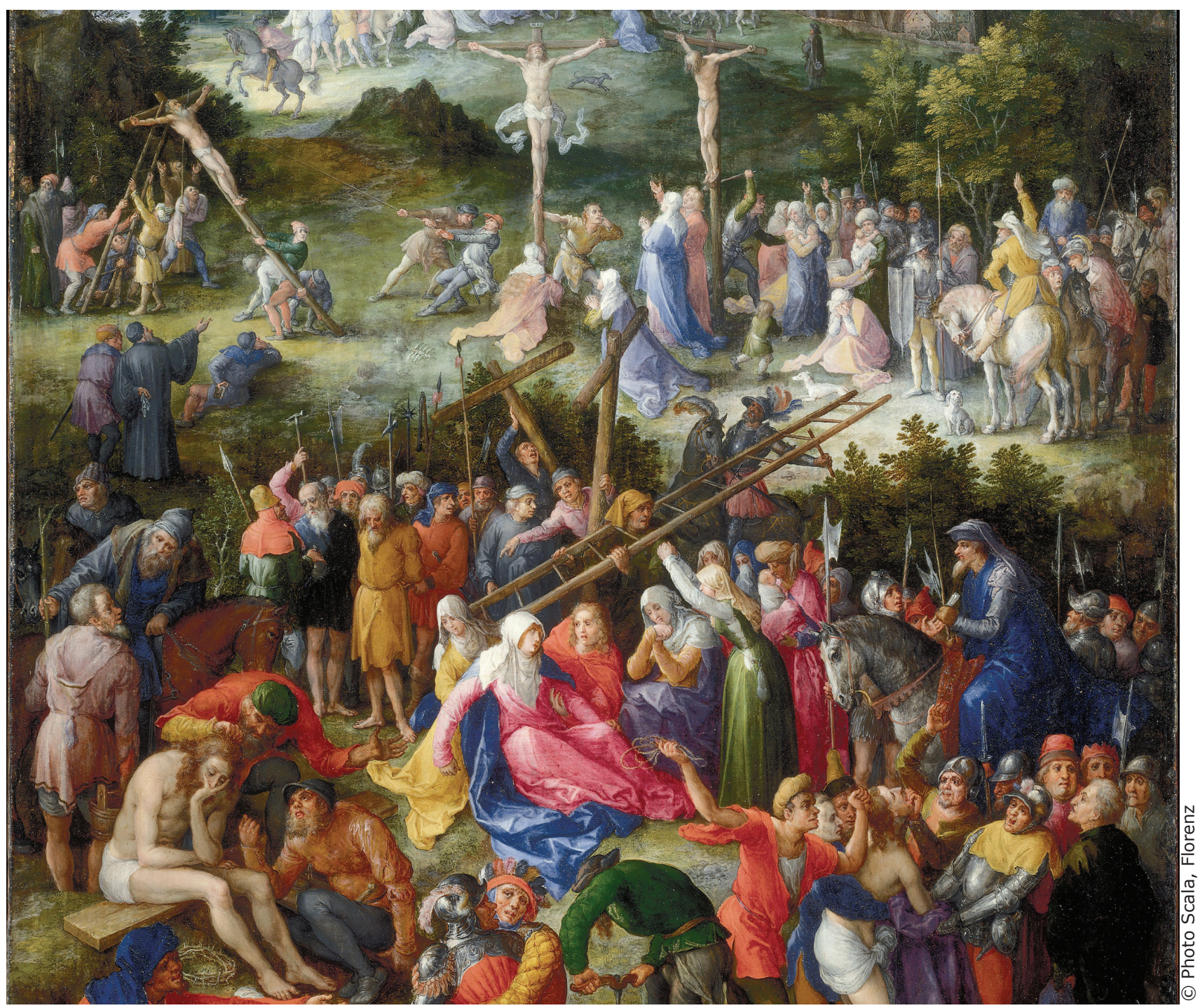

Abb. 5. Detail von Abb. 3.

für den Marienmantel benötigte kostbare Azurit aus dem inzwischen in weiten Teilen vom Osmanischen Reich besetzten Ungarn zu beschaffen. ${ }^{21}$ Die Anekdote macht deutlich, dass es bei der Kopie des berühmten Werkes auch um die Nachahmung der materiellen Wirkungen der Ölfarbe ging. Van Eycks lebendige und lebensähnliche Malerei mache die gemalten Tafeln zu "Spiegeln", so Lucas de Heere in seiner Ode auf den Genter Altar von 1559; der unmittelbar nach der Vollendung von Coxcies Kopie verfasste Text wurde an der Wand gegenüber Van Eycks Retabel platziert und später von Van Mander im Schilder-Boeck im

21 Van Mander 1604, Fol. 200v. Vgl. Dubois 2017, S. 105, Anm. 6; Kemperdick 2014, S. 44.
Wortlaut zitiert. ${ }^{22}$ Auch der spanische Humanist und Sammler Felipe de Guevara hob in den um 1560 verfassten Comentarios de la pintura die "Haltbarkeit" der von Jan van Eyck und anderen niederländischen Künstlern verwendeten Farben hervor, "wie man auf ihren alten Gemälden deutlich sieht". ${ }^{23}$

Im Unterschied zu der im Auftrag von Königin Isabella von Kastilien geschaffenen „Präzisionskopie" von Rogier van der Weydens sogenanntem Miraflores-Altar, die sich erst durch eine dendrochronologische Analyse vom Original unterschei-

22 Van Mander 1604, Fol. 201r: "T'sijn spieghels, spieghels zijnt, neen t'zijn geen Tafereelen."

23 Zit. nach Putzger 2018, S. 115. 


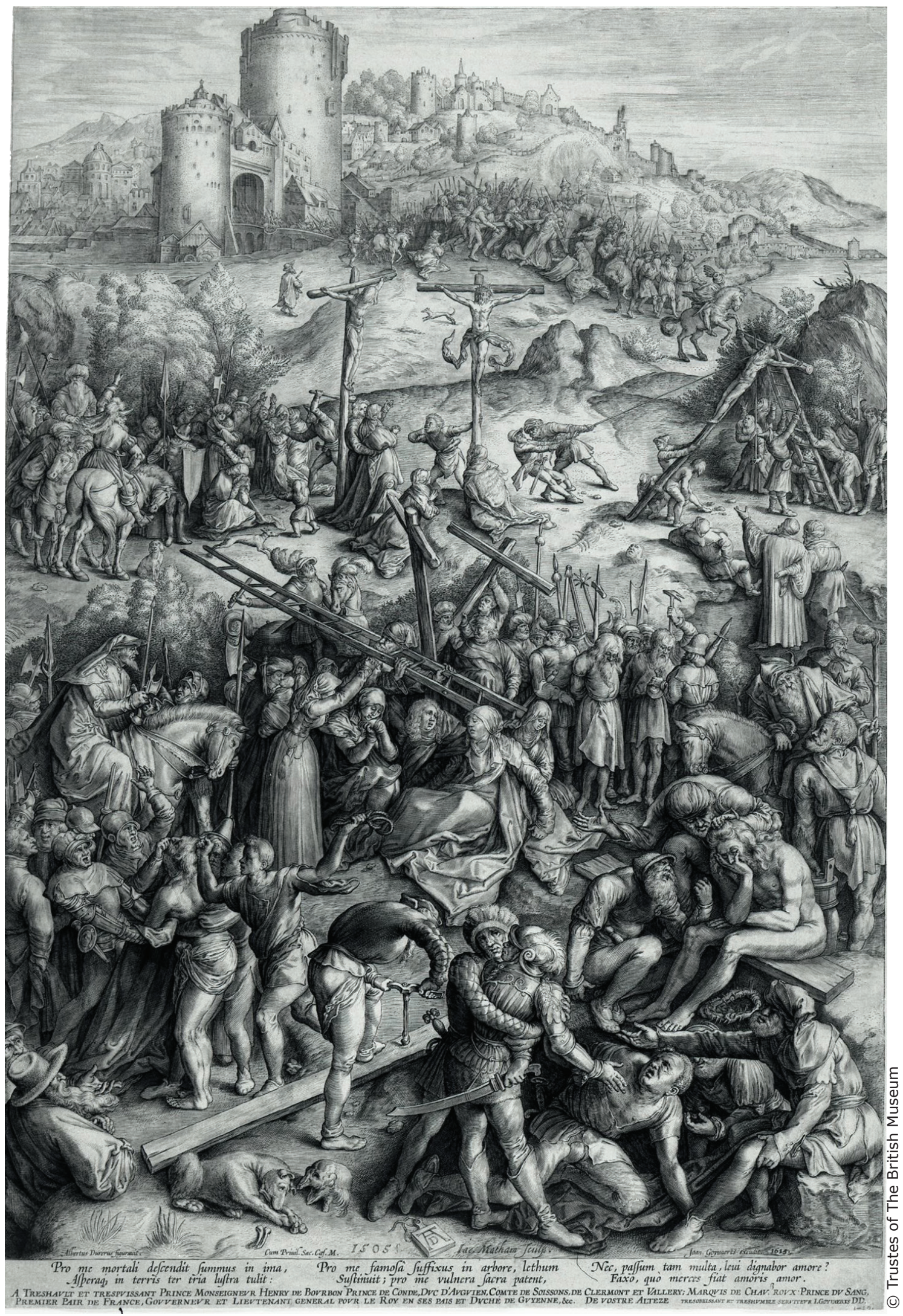

Abb. 6. Jacob Matham nach Albrecht Dürer, Der Große Kalvarienberg, 1615. Kupferstich mit leichter Wasserfarbe, $57,5 \times 39 \mathrm{~cm}$. London, The British Museum, Department of Prints and Drawings, Inv.-Nr. E. 3-180. 


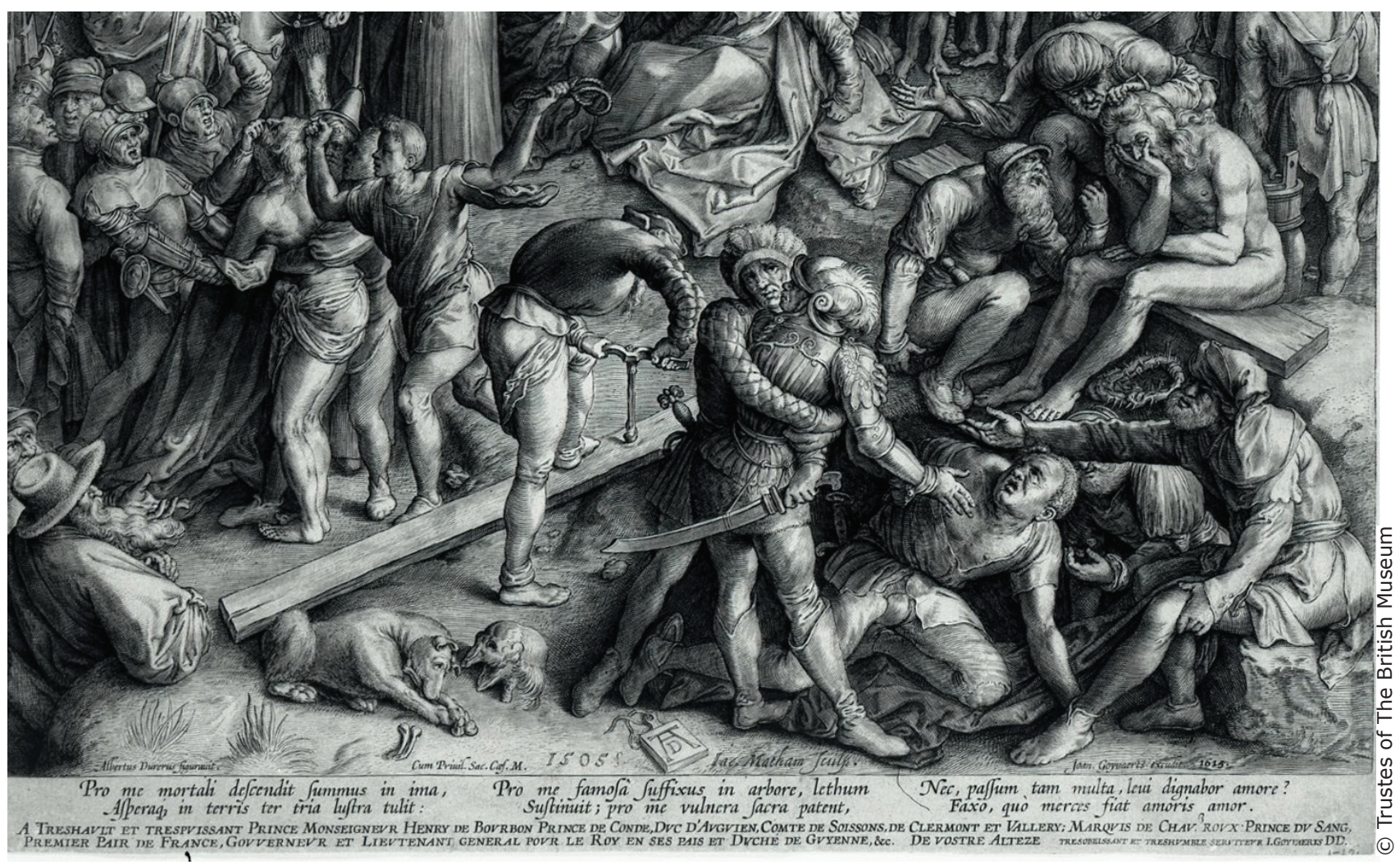

Abb. 7. Detail von Abb. 6.

den lie $\beta,{ }^{24}$ handelt es sich bei Coxcies Kopie des Genter Altars keineswegs um eine exakte Wiederholung. Die Veränderungen der Außenseite, die anstelle der Stifterbildnisse und der zwei Johannesfiguren die Evangelisten zeigt, reagierten auf den neuen Anbringungsort, die Palastkapelle des Alcazar. ${ }^{25}$ Auf der Innenseite ergänzte Coxcie die Gruppe der Streiter Jesu sowohl durch Bildnisse Karls V. und Philipps II., als auch durch ein Selbstporträt mit Lorbeerkranz, bei dem er sich möglicherweise an den angeblichen Porträts der Brüder Van Eyck unter den Gerechten Richtern orientierte. ${ }^{26}$ Die scheinbar vom Bild selbst formulierte Inschrift "Michael de Coxie me fecit anno 155[8?]" nennt einzig den Namen des Kopisten und definiert dadurch die Tafeln als Kopie. Auch De Heere stellte in seiner Ode die Leistungen Van Eycks und Coxcies gleichwertig nebeneinander:

24 Nagel / Wood 2010, S. 275-278. Das originale Triptychon befindet sich in der Gemäldegalerie zu Berlin, die Tafeln der Kopie sind auf das Metropolitan Museum of Art, New York und die Capilla Real, Kathedrale von Granada aufgeteilt.

25 Putzger 2018, S. 110-111.

26 Kemperdick 2014, S. 47 und 55; Putzger 2018, S. 110-111.
Diese "Kopie" ("copie") sei nun in Spanien zu sehen, "als Gedächtnis der Liebe unseres Königs [...] sowie [Van] Eyck und Coxcie zum Ruhm und zur großen Ehre". ${ }^{27}$

Aufschlussreich sind die von Van Mander verwendeten Begriffe. "Copie" (bzw. "copije" oder "kopije") ist im Niederländischen ein seit dem späten 13. Jahrhundert gebräuchliches Wort, das im Etymologicum Teutonicae Linguae von Cornelis Kiel mit "apographum, exemplar exscriptum, exemplum tabulae" übersetzt wird, sich also sowohl auf eine Abschrift als auch auf eine Kopie eines Bildes bzw. ein "Abbild" oder "Ebenbild" bezieht. ${ }^{28}$ Man sucht in Van Manders Schilder-Boeck vergeblich nach dem Wort "originael"; es handelte sich um einen neueren Begriff, der sich erst im Verlauf des

27 Van Mander 1604, Fol. 202r: „In Spaengien dees Copie is (op dat ick't vertelle) / Te Vendedoly nu, tot een ghedachte bloot / Van onses Conings liefde, als die ick boven stelle, / Van Eyck, en oock Coxcy, tot lof en eere groot." 28 Kiel 1599, S. 254 (unter "kopije, wt-schrift"). Vgl. auch die oft zitierte Stelle in Plinius 1997, S. 122-123 (35 XL, 125): "huius tabulae exemplar, quod apographon vocant". 
17. Jahrhunderts etablierte. ${ }^{29}$ Die Bilder und Kunstwerke, welche die Kopien motivierten, bezeichnete Van Mander durchwegs mit dem älteren Begriff des "principael"; ${ }^{30}$ so nannte er auch das von den Brüdern Van Eyck ausgeführte Retabel „principael tafel". ${ }^{31}$ "Principael" (wörtlich: ,der erste') konnte sich gleichermaßen auf ein "Hauptwerk", eine "Hauptperson", eine „Hauptsache" oder, wie das englische "principal", eine "Hauptsumme" oder ein Kapital beziehen, das angelegt oder geliehen werden konnte, folglich Einkommen generierte beziehungsweise Zinsen abwarf. ${ }^{32}$ In diesem Sinne ließe sich auch die Kopie als ,Frucht' eines Hauptwerks oder ,principael' verstehen, die einerseits dessen Wert vergrößert und andererseits wiederum selbst Früchte abwerfen kann.

Der gewinnbringende Handel mit Geld ist nun ein zentrales Thema von Leonardus Lessius' einflussreicher Abhandlung De iustitia et iure libri quatuor (Vier Bücher über Gerechtigkeit und Recht), die erstmals 1605 in Löwen erschien und danach mehrmals neu aufgelegt wurde. ${ }^{33}$ Von zentraler Bedeutung ist das zweite, "der Gerechtigkeit und dem Recht" gewidmete Buch. Das 20. Kapitel "Über Darlehen und Wucher" vergleicht den geliehenen Geldbetrag mit einem "fruchtbaren Saatgut", in welchem "der Gewinn potentiell enthalten" ist, und rechtfertigt die Erhebung von Zinsen als Entgelt für den Verzicht auf den "erhofften oder zu erwartenden Gewinn" beziehungsweise für die berechtigte Furcht vor einem möglichen Schaden. ${ }^{34}$ Das 21. Kapitel „Über Kauf und Verkauf" handelt vom Wert von Waren, die keinen legalen (obrigkeitlich festgesetzten) oder gemeinen (auf einer allgemeinen Einschätzung

29 Vgl. Kiel 1599, S. 712: „originael: archetypus, archetypum, exemplar; authenticae tabulae; primum et principale exemplar".

30 Vgl. Kiel 1599, S. 197: "hoofd penninghen, som-penninghen van den woecker: [...] principalis summa quae foenori datur, vulgo summa capitalis".

31 Van Mander 1604, Fol. 200v.

32 De Marchi / Van Miegroet 1996, S. 32-33.

33 Es gibt eine Fülle von Literatur zu Leonardus Lessius; besonders relevant im Kontext dieses Kapitels: Decock 2009; Baeck 1999.

34 Zitiert nach Van Houdt 1999, S. 114; Lessius 1999 [1605], S. 234 (liber secundus, sectio tertia, cap. 20, dubitatio 11 [80]): "[...] pecunia illa [...] est enim veluti semen foecundum lucri per industriam, in quo lucrum ipsum virtute continetur: ergo plus pro ea exigi potest, quam ipsa per se valeat." beruhenden) Preis haben, wobei als Beispiele "Edelsteine, herausragende Hunde, Falken, ,indische' Vögel und alte Bilder" genannt werden, folglich jene Güter, die sich etwa in fürstlichen Kunstkammern und Menagerien finden; deren Preis sollte sich aus "der Einschätzung kenntnisreicher Menschen oder wenigstens gemäß der Einschätzung des Verkäufers selbst" ableiten, "der in guter Absicht alle relevanten Umstände erwogen hat". 35 Allerdings konnte die "persönliche Wertschätzung", die ein Besitzer für bestimmte Dinge empfand, in die Preisberechnung mit einbezogen werden. ${ }^{36}$ Gerade der letzte Punkt war für den von konkurrierenden Händlern und Agenten geprägten frühneuzeitlichen Kunstmarkt von besonderem Interesse.

\section{Kopieren als Verlebendigen}

Im Schilder-Boeck von 1604 empfiehlt Karel van Mander denjenigen, die das Neueste und Beste an gegenwärtiger (flämischer und deutscher) Kunst sehen wollten, eine Reise nach Prag. ${ }^{37}$ Es befanden sich damals acht der besten Werke von Pieter Bruegel dem Älteren im Besitz des Kaisers, wie Van Mander im Leben des Künstlers erwähnt. ${ }^{38}$ Jan Brueghel selbst hatte sich vergeblich bemüht, für den Mailänder Kardinal Federico Borromeo ein Werk seines Vaters zu beschaffen. Da der Kaiser keine Ausgaben gescheut habe, um möglichst alle Werke zu erwerben, sei keines mehr

35 Zitiert nach Van Houdt 1999, S. 121-122; Lessius 1999 [1605], S. 253 (liber secundus, sectio tertia, cap. 21, dubitatio $3[15-16])$.

36 Zitiert nach Van Houdt 1999, S. 253; Lessius 1999 [1605], S. 254 (liber secundus, sectio tertia, cap. 21, dubitatio 3 [17]): „Utrum res eae, quae non habent pretium legitimum, vel vulgare (ut sunt quaedam gemmae, insignes canes, falcones, aves indicae, picturae veteres, \& simila) possint vendi quanti volet dominus [...] Respondeo tamen, contrarium verius videri, nempe res tales non posse vendi pro arbitrio venditoris, sed iuxta aestimationem intelligentium, vel certe iuxta aestimationem ipsius venditoris, bona fide spectatis cirumstantiis supradictis."

37 Van Mander 1604, Fol. *4v.

38 Van Mander 1604, Fol. 233v. Van Mander erwähnt eine größere und eine kleinere Version des Turmbaus zu Babel, zwei Versionen der Kreuztragung, den Betlehemitischen Kindermord, eine (nicht identifizierbare) Versuchung Jesu und die Dulle Griet. 


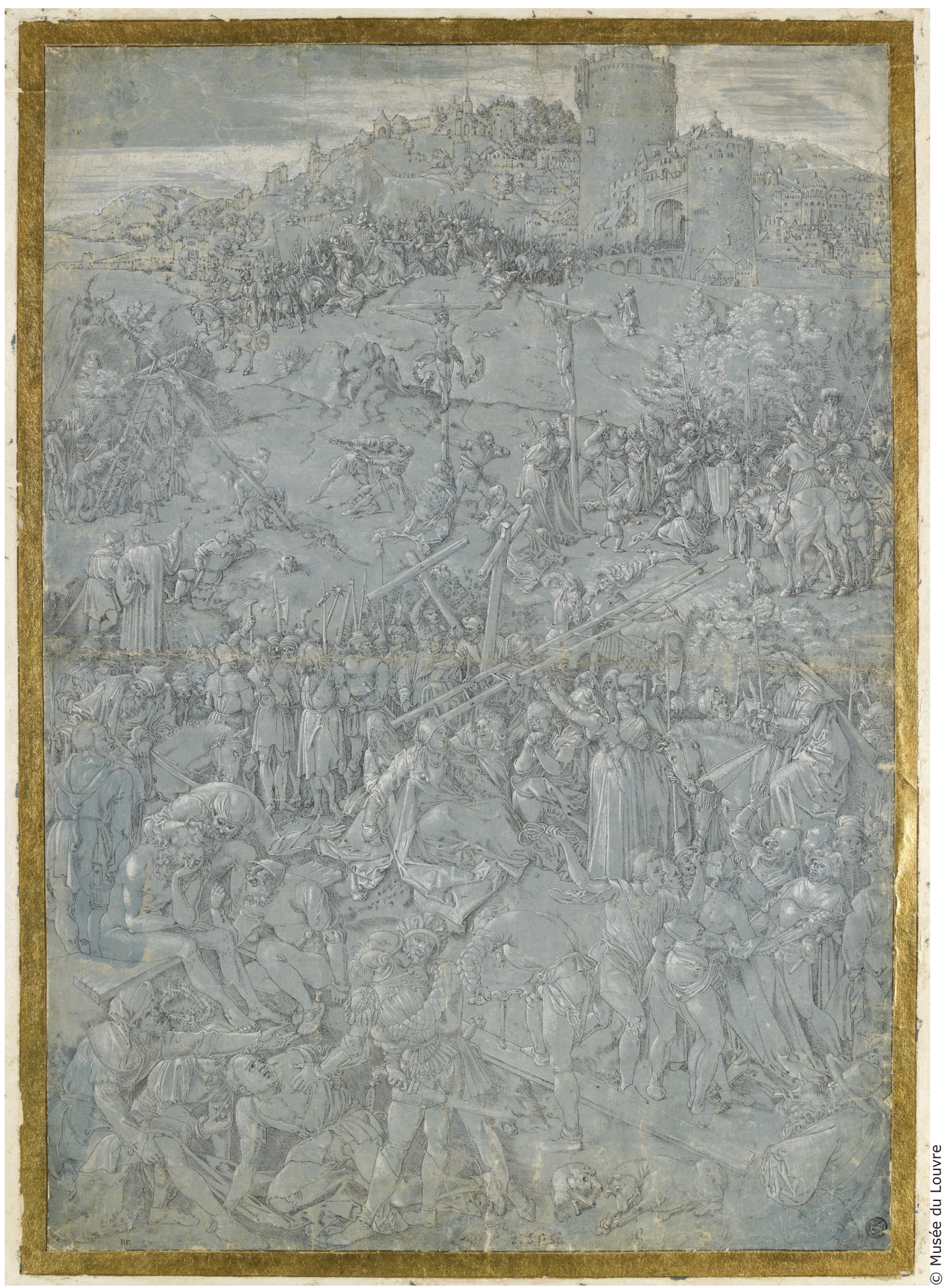

Abb. 8. Nach Albrecht Dürer, Der Große Kalvarienberg. Federzeichnung auf grün grundiertem Papier, weiß gehöht, 55,6 x $39 \mathrm{~cm}$. Paris, Musée du Louvre, Cabinet des dessins, Inv.-Nr. 18.640. 
zu kaufen. ${ }^{39}$ Rudolf II. besaß auch die damals größte Sammlung von Dürers Werken, einschließlich einer Vielzahl seiner Zeichnungen. Er gehörte zu den ersten Kunstliebhabern, die sich für Zeichnungen interessierten. Aus dem noch zu Lebzeiten des Kaisers angelegten Inventar geht hervor, dass die Zeichnungen und Graphiken, wie ein Großteil der anderen Objekte, in Truhen und anderen Behältern aufbewahrt wurden. ${ }^{40}$ Die Kunstkammer diente dabei nicht ausschließlich der Repräsentation, sondern vielmehr auch der künstlerischen und wissenschaftlichen Forschung, die wiederum politisch und territorial genutzt werden konnte. ${ }^{41}$ Sie war als ,Studiensammlung' angelegt, welche die direkte Auseinandersetzung mit den Objekten (die neben Zeichnungen, Drucken und Gemälden auch Uhren, wissenschaftliche Instrumente sowie eine Vielzahl von Kunst- und Naturobjekten aus allen Teilen der Welt umfassten) ermöglichte. Das Kopieren und Übertragen von Zeichnungen schärfte den Blick für unterschiedliche ästhetische Verfahren. Wie Andrea Bubenik gezeigt hat, benutzten in Prag oft mehrere Künstler dieselbe Vorlage Dürers, um diese in unterschiedliche Medien und Materialien zu übertragen. ${ }^{42}$ So fertigte etwa der Antwerpener Stecher Aegidius Sadeler 1597 und 1598 mehrere Kupferstiche nach Dürers Kopfstudien an, mit denen sich schon Hans Hoffmann in den 1580er Jahren zeichnerisch auseinandergesetzt hatte. ${ }^{43}$ Sadeler übertrug Dürers Kompositionen jeweils spiegelbildlich auf die Kupferplatte, so dass der Druck gegenüber der Vorlage nicht seitenverkehrt erschien. Eine Inschrift auf einer dreidimensional gestalteten Tafel, wie sie sich auch in Dürers Porträtkupferstichen findet, nennt das Jahr, in dem "der Deutsche Albrecht Dürer" das Bildnis geschaffen ("fecit"), sowie jenes, in dem Aegidius Sadeler es "gestochen" hat ("scalpsit"), definiert folglich die Kopie als ein gemeinschaftliches Werk, das zudem zeitlich fast ein Jahrhundert auseinan-

39 Koerner 2016, S. 90; Crivelli 1868, S. 119 (Brief vom 6.2.1609): "Sin a hora ha cercate un quader del mio pader, per mandare a VS III.mo, ma non trove niente a proposite. L'imperator ha fatto gran spese per aver tutti sua opera."

40 Bauer / Haupt 1976, S. 130-139 (Bücher, Graphiken, Zeichnungen).

41 Reitz 2015, S. 63-80, 536-550; Bukovinská 2007; Bukovinská 2003; Bukovinská 1986; Fučíková 1986.

42 Bubenik 2013, S. 103-136.

43 Bubenik 2013, S. 105-107. der liegende Stilqualitäten und Techniken vereint (Abb. 10).

Jan Brueghel der Ältere trat im Frühjahr 1604 eine achtmonatige Reise an, die ihn über Holland, Zeeland und Frankfurt nach Prag führte, just in dem Jahr, in dem auch Van Manders SchilderBoeck erschien. ${ }^{44}$ Im Schilder-Boeck findet sich auch ein kurzer Eintrag zu dem damals 36-jährigen Künstler, der sich in den frühen 1590er Jahren über längere Zeit in Neapel, Rom und Mailand aufgehalten hatte und dessen Werke im Kreis aufstrebender junger Kleriker in Rom, zu denen auch Kardinal Federico Borromeo zählte, auf großes Interesse stieß. Van Mander erwähnt, dass sich Brueghel mit seinen "Landschaften und sehr kleinen Bildchen", bei denen er eine "wunderbar erfindungsreiche Art" an den Tag legte, eine große Achtung erworben hatte. ${ }^{45}$

Brueghel hatte sich schon in einem auf Kupfer gemalten, 1598 datierten Kalvarienberg mit Dürers Komposition auseinandergesetzt, die ihm damals vermutlich in einer gemalten Kopie vorlag; die nach Dürer zitierten Bildelemente bereitete er durch Unterzeichnungen vor, die sich in seinen Gemälden sonst nur selten finden. ${ }^{46}$ Die verkleinerten figürlichen Zitate nach Dürer sind hier bloße Staffage einer horizontal angelegten Landschaft; Heilsgeschichte entfaltet sich in der Dramatik eines als Schädelstätte gekennzeichneten gebirgigen Ortes unter einem düsteren, sich allmählich verdunkelnden Himmel. Brueghels gemalte Kopie von Dürers Zeichnung, die er im Sommer 1604 vor dem Original am Prager Hof ausführte, wurde höchstwahrscheinlich

44 De Clippel / Van der Linden 2015, S. 84-85. Jan Brueghel der Ältere reiste mit einem von Erzherzog Albert von Österreich für acht Monate ausgestellten Reisepass: Brueghels Aufenthalt in Prag ist weiter durch die Signatur einer Zeichnung dokumentiert: "BRVEGHEL fecit in praga 1604". Jan Brueghel der Ältere, Waldlandschaft mit Straße, 1604. Feder und braune Tinte, braun und blau gewaschen, 230 $x 194 \mathrm{~mm}$. The British Museum, Department of Prints and Drawings, Inv.-Nr. 1853.0813.44.

45 Van Mander 1604, Fol. 234r: „Hy reysde voort nae Colen / en soo in Italien / en is in seer groot achten ghecomen / met te maken Landtschapkens / en seer cleen beeldekens / daer hy een uytnemende fraey handelingh van heeft."

46 Jan Brueghel der Ältere, Der Kalvarienberg, 1598. Kupfer, $36,3 \times 55,2 \mathrm{~cm}$. München, Bayerische Staatsgemäldesammlungen, Alte Pinakothek, Inv.-Nr. 823. Neumeister / Ortner / Schmidt 2013, S. 95; Neumeister 2013, S. 200-203, Kat. 26; Ertz / Nitze-Ertz 2008-2010, Bd. 2, S. 587-589, Kat. 274. 


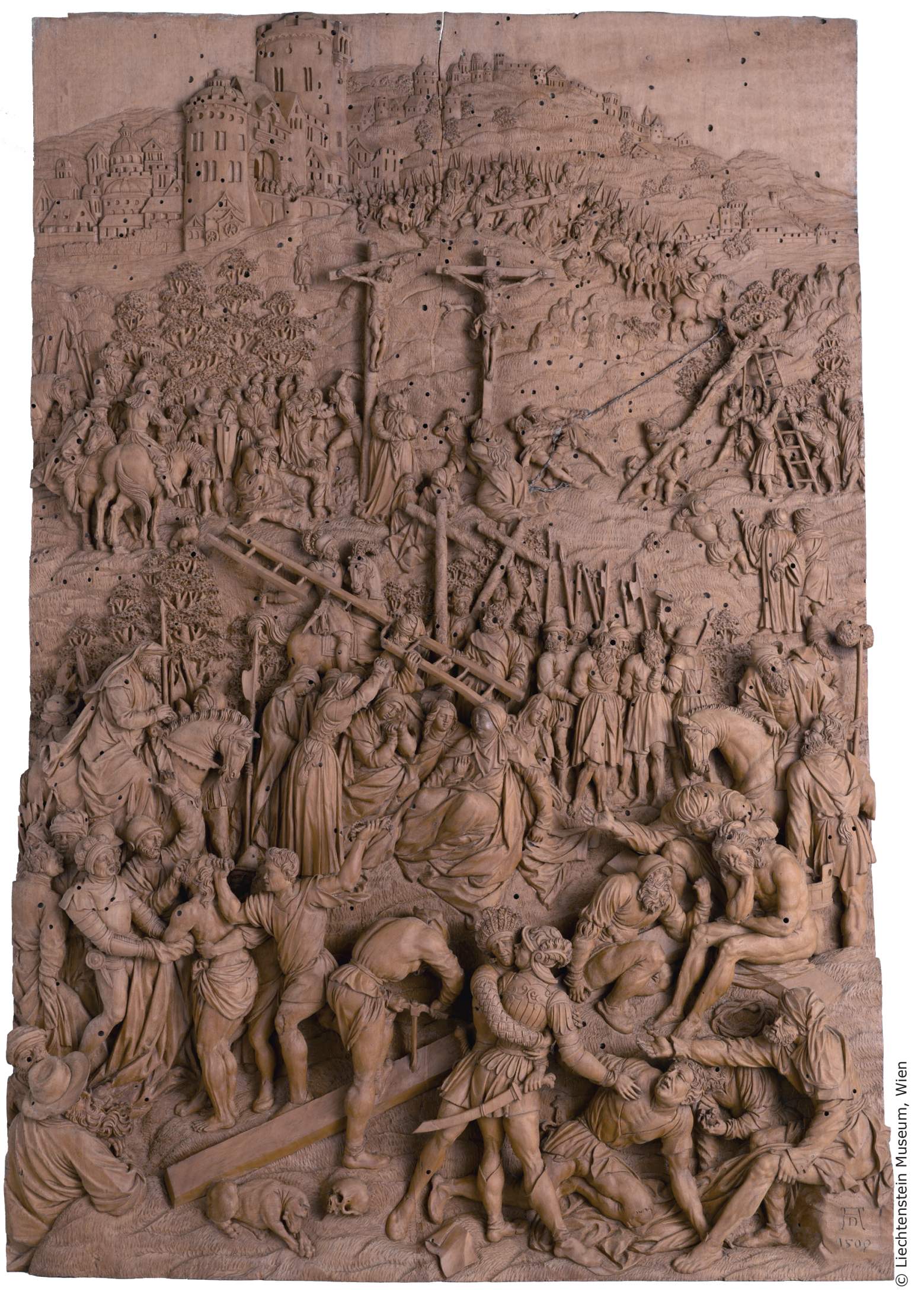

Abb. 9. Unbekannter Bildschnitzer nach Albrecht Dürer, Der Große Kalvarienberg, nach 1615 . Relief, Holz, 55 x 37.8 cm. Wien, Liechtenstein Museum, Inv.-Nr. 618. 
direkt von Rudolf II. in Auftrag gegeben (Abb. 3, $4,5) .{ }^{47}$ In dessen Augen bestand folglich eine Verwandtschaft oder Anziehungskraft zwischen Dürers monochromer und Jan Brueghels bunter Malerei. In welcher Weise artikulierte Brueghel in der malerischen Neubearbeitung von Dürers Zeichnung auch seine eigenen künstlerischen Anliegen? Brueghel überführte Dürers berühmte Linienführung in das Idiom kleinfiguriger Ölmalerei. Darüber hinaus fügte er Dürers monochromer Zeichnung Glanz und Farben hinzu - jene Qualitäten, die nach der frühneuzeitlichen Traktatliteratur einer Darstellung Lebendigkeit, Bewegung und Präsenz verleihen ${ }^{48}$ - und erneuerte dadurch die affektive Wirkung, die man an Dürers ,Kunst des Einfarbigen' lobend hervorhob. In den Kapiteln zur Farbe in Den grondt der edel vry schilder-const (Grundlage der edlen und freien Malerkunst), dem in Versen verfassten ersten Band des Schilder-Boeck, vergleicht Van Mander das Zeichnen mit dem Körper, die Malerei jedoch mit der Seele: "Denn durch die Farbe erhalten die toten Striche der Zeichnung Leben und Bewegung und wird ihnen die rechte Auferweckung gegeben." ${ }^{\prime 49}$

In der vergleichenden Gegenüberstellung der zwei zu unterschiedlichen Zeiten und in verschiedenen Medien geschaffenen Kunstwerke Dürers und Brueghels traten deren Qualitäten umso deutlicher hervor. Während bei Dürer die affektive Gestaltung der Mimik und Gestik der am Passionsgeschehen beteiligten Figuren im Vordergrund stand, erzeugte Brueghel Dramatik durch eine stärkere Betonung landschaftlicher Texturen sowie eine wechselvolle Beleuchtung der unterschiedlichen Schauplätze. In Dürers Zeichnung und Brueghels Gemälde nimmt die Erzählung im Hintergrund mit der Ankunft Jesu auf Golgatha ihren Anfang, wird dann im Vordergrund weitergeführt und endet im Mittelgrund mit der

47 Brueghels benutzte eine Tafel von leicht größerem Format als Dürers Zeichnung. Zu Brueghels Gemälde: Honig 2016, S. 20; Neumeister 2013, S. 204-205, Kat. 27 (Francesca de Luca); Hirakawa 2009, S. 119-135; Silver 2008; Ertz / Nitze-Ertz 2008-2010, Bd. 2, S. 590-594, Kat 275; Bedoni 1983, S. 132, 183-184; Bodart 1977, S. 88-89, Kat. 20; Lossky 1959.

48 Zur Farbe und zum Relief als Mittel der Evidenzerzeugung: Von Rosen 2000, S. 184-186. Zur Animationskraft der Farbe: Fehrenbach 2005; Dackerman 2002.

49 Van Mander 1604, Fol. 46v: „Want door verwen worden de doode streken / Der teyckeninghen te roeren en leven / En de rechte verweckinghe ghegheven."
Kreuzigung. In Brueghels Gemälde sind die einzelnen Episoden der Passionsgeschichte zudem mit Farbe, Licht und Schatten voneinander unterschieden. Sowohl der Hintergrund als auch der Mittelgrund sind in vorwiegend blassen, subtilen Farbtönen gemalt. Die bekannten Szenen der Kreuztragung und der Kreuzigung heben sich unter dem sich verdunkelnden Himmel nur schwach von der Landschaft ab. Im Gegensatz dazu wird im Vordergrund zwei selten dargestellten Szenen der Passion durch rote, orangerote und gelbe Töne Farbe und Leben verliehen: der Entkleidung Jesu und seinem geduldigen Warten auf die Annagelung an das Kreuz. Brueghel übernahm auch Dürers Paare von bildinternen Zeugen, wobei durch die Farbe der Kleidung angezeigt wird, welchem Teil der Heilsgeschichte sie angehören. Die Hüte und spitzen Bärte der in rote Gewänder gehüllten Figuren in der rechten unteren Ecke identifizieren sie als Juden, die sich in ihrer Ambivalenz auch auf die jeweiligen Betrachter beziehen, die durch ihre Sünden Jesu Passion fortwährend erneuern. Zwei weitere, blau akzentuierte Zeugen erscheinen in der oberen Hälfte der Komposition nahe am linken Bildrand; während der eine mit der Hand auf Jesus am Kreuz hinweist, dreht der andere seinen Kopf, um nach hinten zu blicken. Diese Figuren artikulieren die Wechselbeziehung zwischen Betrachter und Geschehen im Bild, zwischen Golgatha-Hügel, Kunstkammer und dem in der Imagination konstruierten Einsamkeitsort der Passionsmeditation.

Es wurde in der Forschungsliteratur bisher wenig beachtet, dass Jan Brueghel, wohl nach dem Vorbild der Kupferstiche des nahezu gleichaltrigen Aegidius Sadeler, sowohl Dürers Erfindung als auch seine eigene Schöpfung und Ausführung auf der rauen Oberfläche des Steins links im Vordergrund dokumentiert hat, auf welchem der Mann mit der blauen Kopfbedeckung eindringlich seinen Anteil an der Gewandung Jesu einfordert (Abb. 4). Die ausholende Geste der auffälligen Figur unterstreicht Brueghels Anspruch auf seinen Anteil am Gemälde. Die erste Zeile der Inschrift zeigt Dürers Monogramm - ein großes A mit einem eingeschriebenen $D$ - gefolgt vom Wort "INVENTOR" und der Jahreszahl 1505, die zweite Zeile lautet „BRUEGHEL FEC[IT] 1604”. Es handelt sich dabei um eine für ein Gemälde ungewöhnliche Künstlerinschrift, welche die geteilte Autorschaft zwischen ursprünglichem Erfinder 


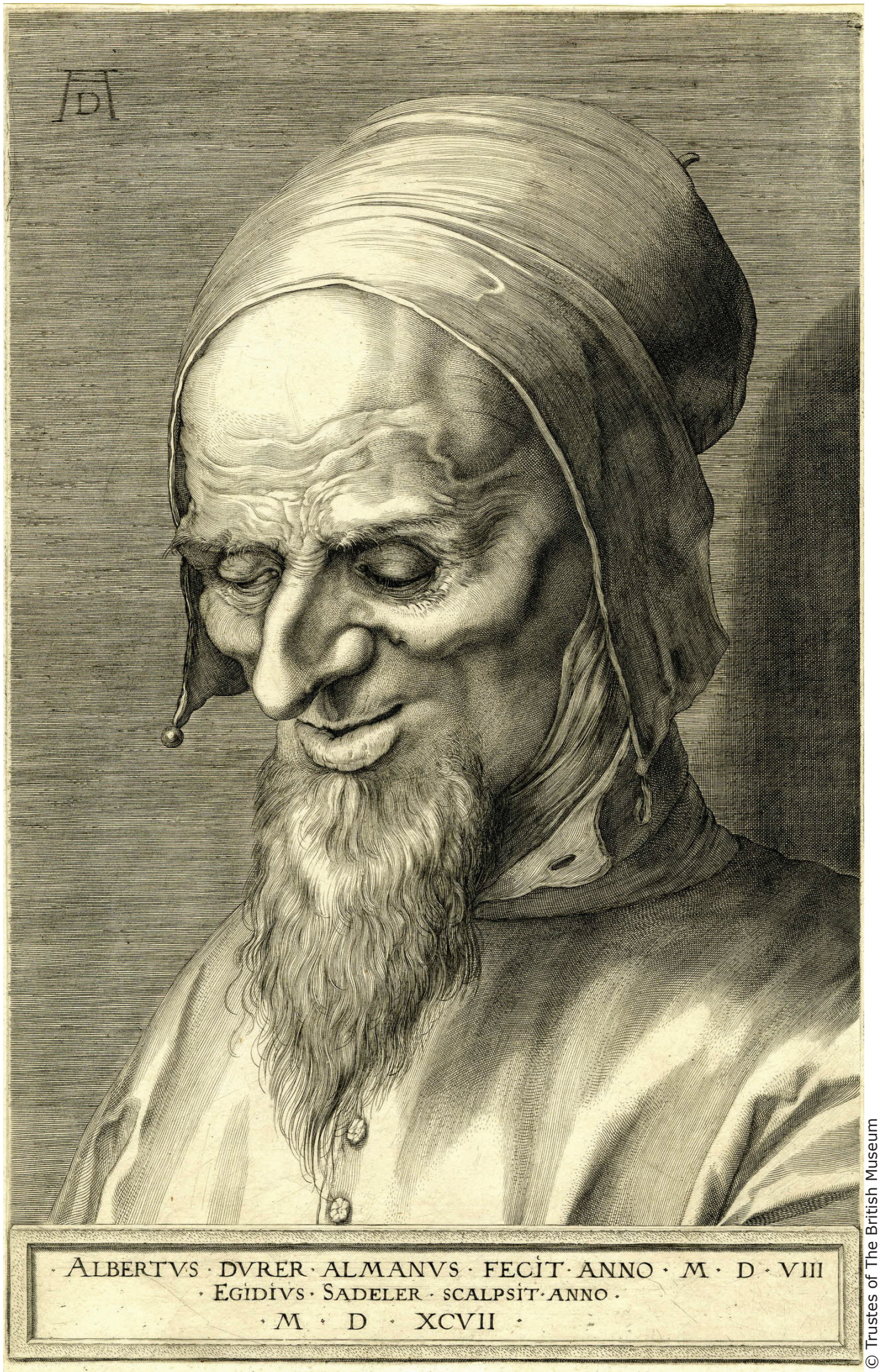

Abb. 10: Aegidius Sadeler nach Albrecht Dürer, Kopf eines Apostels, 1597. Kupferstich, 35,8 x 22,9 cm. London, British Museum, Inv.-Nr. E. 3-186. 
und neuem Schöpfer zum Ausdruck bringt und dabei auch markiert, dass zwischen der Erfindung und Neufindung der Komposition eine Zeitspanne von genau 99 Jahren liegt. Bemerkenswerterweise hat Brueghel Dürers Monogramm von seiner ursprünglichen Position auf dem Täfelchen im mittleren Vordergrund in die Nähe seiner eigenen Signatur gerückt und dadurch sich selbst in die Nachfolge Dürers gestellt.

Wie Tobias Burg nachweisen konnte, waren die Niederlande und vor allem Antwerpen an der Erfindung und Verbreitung von Künstlersignaturen in der Frühen Neuzeit entscheidend beteiligt. Pieter Bruegel der Ältere signierte nach Burg ,fast drei Viertel $(70 \%)^{\prime \prime}$ seiner Werke. ${ }^{50}$ Darüber hinaus haben sich einzelne Maler bei der Anbringung inrer Signatur auf Gemälden von Signaturen der Antwerpener Druckgraphik anregen lassen, also jener Kunst, in der Antwerpen in der Mitte des 16. Jahrhunderts besondere Berühmtheit erlangte. So signierte etwa der Antwerpener Maler Frans Floris mehrere Werke mit dem sonst nur in der Druckgraphik gebräuchlichen Ausdruck „invenit et fecit". ${ }^{51}$ Wie Dürers Monogramm wurde auch die Signatur von Hieronymus Bosch häufig von anderen Künstlern appropriiert: Der von Pieter van der Heyden nach einer Zeichnung von Pieter Bruegel dem Älteren verfertigte Kupferstich Große Fische fressen kleine Fische trägt die Inschrift "Hieronijmus Bos inventor"; Bruegel selbst, der zu seiner Zeit auch als "zweiter Hieronymus Bosch" und Erneuerer seiner Kunst gehandelt wurde, wird nicht genannt. ${ }^{52}$ Der Verweis auf die Autorschaft Boschs diente einerseits dem Verkaufsinteresse des Verlegers Hieronymus Cock, legte aber auch künstlerische Verwandtschaften offen. Jan Brueghels eigene Signatur orientierte sich an derjenigen seines Vaters, der anfänglich in niederländischer Schreibweise mit "brueghel" signierte, dann eine latinisierte Schreibweise in römischen Kapitallettern entwickelte, die auf das $\mathrm{H}$ verzichtete. ${ }^{53}$ Jan Brueghel behielt in seiner Signatur den Buchstaben $\mathrm{H}$ bei, wohl auch um seine Autorschaft von derjenigen seines berühmten Vaters

50 Burg 2007, S. 400.

51 Burg 2007, S. 424.

52 Zur Entstehungsgeschichte des Kupferstichs: Koerner 2016, S. 81-83.

53 Burg 2007, S. 434-436; Neumeister 2013, S. 429-431 ("Übersicht der Signaturen”). zu unterscheiden. Die formale Angleichung der Signatur an diejenige seines Vaters diente nicht zuletzt auch einer geschickten künstlerischen Selbstvermarktung.

In Brueghels kreativer Anverwandlung einer zentralen religiösen historia zeigte sich seine Affinität für Dürers graphisches Werk und für Dürer als Künstler. Wie Jan van Eyck wurde auch Dürer in den Niederlanden um 1600 zur entscheidenden Referenzperson für die Geschichte der eigenen künstlerischen Tradition. Verband man mit Van Eyck die ,Erfindung' der Ölmalerei, die als ,Neugeburt der Malerei' rezipiert wurde, so wies Dürer einen neuen Weg, um in der Graphik Möglichkeiten und Grenzen der Darstellbarkeit zu erkunden. 1549 ließ die Antwerpener Malergilde einen Prunkbecher mit den Bildnissen Apelles', Zeuxis', Raffaels und Dürers anfertigen. ${ }^{54} 1563$ schmückte der Maler und Kaufmann Cornelis van Dalem sein Haus an der Lange Nieuwstraat in Antwerpen mit einer Allegorie der Malerei, die von den Porträtbüsten Dürers und Van Eycks flankiert war, die sich bis heute erhalten haben. ${ }^{55}$

\section{Geteilte Autorschaft}

Brueghels Ölgemälde von 1604 diente nicht als ,Ersatz' für Dürers Feder- und Pinselzeichnung von 1505, sondern vielmehr als deren unzertrennliches Pendant, das mit den entwickelten Mitteln der Malerei das unübertreffliche Vorbild Dürers zu erneuern versuchte. Im frühen 17. Jahrhundert wurden die beiden Werke als Bilderpaar aufgefasst und gelangten auch gemeinsam als Geschenk nach Florenz, wo sie noch heute in den Uffizien zu sehen sind. Nach einem auf den 28.12.1628 datierten Eintrag in einem mediceischen Inventar befand sich das Bilderpaar in der Tribuna; der Eintrag vermittelt ein sehr genaues Bild über die Art der Aufbewahrung der beiden Werke, die in aller Wahrscheinlichkeit schon in Prag angeordnet worden war. ${ }^{56}$

54 Göttler / Meganck 2015, S. 343-344.

55 Göttler / Meganck 2015, S. 342-343.

56 Zitiert nach Barocchi / Gaeta Bertelà 2005, S. 74-75, Anm. 281 (Inventario di tutte le figure, quadri et altre cose della tribuna, 1603-1631: Archivio della Biblioteca degli Uffizi, Ms. 71, c. 56): "Un quadro in dua parte mastiettato in mezzo che si apre con cornice d'ebano, alto braccia 1 1/5, 
Nach der Beschreibung handelte es sich um ein aus zwei in Ebenholz gerahmten Tafeln bestehendes "Bild" ("quadro"), das mittels eines Scharniers geöffnet werden konnte; bei geöffnetem Zustand war auf der einen Seite Dürers chiaroscuro Zeichnung des Kalvarienbergs mit einer "Vielzahl von Figuren", auf der anderen Seite Brueghels "gemalte Kopie derselben" ("la copia di esso colorito") zu sehen. Die schützende Außenseite (die ,Rückseite' von Dürers Zeichnung) zeigt noch heute eine Landschaft mit dem Blick auf eine Stadt am Fluss. Im Vordergrund sind eine Frau mit einem Korb, eine Gruppe von Männern, ein Hund und mehrere Ziegen zu sehen. ${ }^{57}$ Weiter wird vermerkt, dass die zwei Tafeln Maria Magdalena von Österreich (1589-1631), der Gattin von Cosimo II. de' Medici (1590-1621), von ihrer Mutter, Maria Anna von Bayern (1551-1608), geschenkt wurden. Maria Anna von Bayern war die Gattin von Erzherzog Karl II. von Innerösterreich (1540-1590), einem Onkel von Rudolf II. Möglicherweise gelangten die beiden Tafeln 1608 aus Anlass der Hochzeit von Cosimo II. de' Medici und Maria Magdalena nach Florenz. ${ }^{58}$

Diese Art der Aufbewahrung schützte Dürers Zeichnung einerseits vor weiteren Beschädigun-

largo braccia $4 / 5$, ch'è in una parte di dentro un disegno di minio di chiaroscuro d'Alberto Duro, del Monte Calvario con Nostro Signore in croce e più quantità di figure significanti quando I'andorno a crucifigere e da l'altra banda la copia di esso colorito di mano del Bruces e per di fuori, nella parte dinanzi un paesino con figure e animali con un cespuglio di un quercione, similmente colorito dal detto Bruces. Donato a Sua Altezza Serenissima dalla Serenissima Arciduchessa sua madre." Vgl. auch Gaetà Bertelà 1997, S. 69, Nr. 751 und Nr. 752 und Anm. 481. Ein Hinweis auf diese frühen Dokumente findet sich im Katalogeintrag von Francesca de Luca (Neumeister 2013, S. 204, Kat. 27). Hirakawa 2009, S. 119-135, und Bedoni 1983, S. 183-184, verweisen lediglich auf die Beschreibungen von Franceso Zacchiroli (Description de la Galerie Royale de Florence, 1783) und Luigi Lanzi (La Real Galleria di Firenze, 1782).

57 Öl auf Holz, 56 x 38,5 cm. Florenz, Galleria degli Uffizi, Gabinetto dei Disegni e delle Stampe, Inv.-Nr. 1890 n. 8406. Bodart 1977, S. 90-91, Kat. 21 (mit falschen Maßangaben); Ertz / Nitze-Ertz 2008-2010, Bd. 2, S. 577-580, Kat. 270 (mit falschen Maßangaben). Mein herzlicher Dank geht an Luciano Mori, Gabinetto dei Disegni e delle Stampe, für seine Hilfe bei der Ausmessung der Tafel und der Bestimmung des Mediums.

58 Zwischen 1602 und 1605 war Maria Magdalena öfters auch als mögliche Ehefrau Kaiser Rudolfs II. im Gespräch. Zu Maria Annas Heiratsprojekten für Maria Magdalena: Keller 2012, S. 187-189. gen und verlegte sie andererseits in einen intimeren und zugleich exklusiveren Kontext. Nun wurden schon im Venedig des 16. Jahrhunderts chiaroscuro Zeichnungen in Kästen aufbewahrt, wie etwa aus einem Inventar des Kunstbesitzes der Familie Vendramin hervorgeht. ${ }^{59}$ Dass eine Zeichnung gemeinsam mit ihrer gemalten Kopie aufbewahrt wurde, war jedoch neu und muss in Verbindung mit den Kopierpraktiken am Prager Hof gesehen werden: Brueghels Ölgemälde diente gewissermaßen als visuelles Dokument der Neubelebung von Dürers Kunst durch einen flämischen Meister im hundertsten Jahr nach der Fertigstellung von Dürers berühmtem Werk. Es bot Dürers Zeichnung einen neuen interpretativen und meditativen Rahmen, in dem sie betrachtet und verstanden werden konnte.

Bemerkenswerterweise wurde Dürers Zeichnung auch in Haarlem rezipiert, Van Manders Wahlstadt, die sich im frühen 17. Jahrhundert in Konkurrenz zu Antwerpen zu einem neuen Zentrum der Künste entwickelte. Das von Jacob Matham gestochene Blatt nach Dürers Zeichnung (oder nach einer Kopie nach Dürers Zeichnung) wurde 1615 vom Drucker Joannes Goyvaerts herausgegeben (Abb. 6, 7). Nachdem Goltzius sich nach 1600 zunehmend der Malerei zugewandt hatte, avancierte Matham zum bedeutendsten Stecher Haarlems, dem Rudolf II. zudem 1601 die kaiserlichen Druckprivilegien verlieh. ${ }^{60}$ Es ist schon vermutet worden, dass sich Matham an der exakten, ebenfalls auf grün grundiertem Papier ausgeführten Kopie orientiert hat, die sich heute im Louvre befindet (Abb. 8). ${ }^{61}$ Im unteren Teil des Stichs befindet sich an prominenter Stelle das Täfelchen mit Dürers Monogramm sowie dem Datum 1505. Die Inschrift zeigt an, dass Dürer die Komposition formulierte (figuravit), Matham sie stach (sculpsit) und Goyvaerts sie 1615 publizierte (excudit) (Abb. 7). Sie verdeutlicht den langen Prozess der Entstehung, der in diesem Fall nicht in Antwerpen oder Prag, sondern im Haarlem von Goltzius und Van Mander ein vorläufiges Ende nahm.

59 Windows 2012, S. 34.

60 Widerkehr 2007, Bd. 1, S. 82-84, Nr. 39; Bartrum 2002, S. 279-280, Kat. 238.

61 Filedt Kok 1996, S. 339; Demonts 1937, Bd. 1, S. 36-37, Nr. 185, Tafel LXIII. Die Zeichnung stammt aus der bedeutenden Sammlung des Kölner Handelskaufmanns Everhard Jabach (1618-1695), der vielfältige Beziehungen zu den Niederlanden hatte. 
Eine von Van Mander überlieferte Anekdote unterstreicht die zentrale Bedeutung Dürers für Goltzius' Selbstverständnis als Kupferstecher und Zeichner. Goltzius hatte seinen von einem Holzschnitt Dürers angeregten Kupferstich der Beschneidung Jesu mit einem Selbstporträt und einem ebenfalls an Dürer orientierten mittig platzierten quadratischen Täfelchen versehen, auf dem jedoch sein eigenes Monogramm HG und die Jahreszahl 1594 erscheint. ${ }^{62}$ Nach Van Mander soll nun Goltzius auf der Frankfurter Messe das Porträt und das Monogramm herausgebrannt, den Stich mit Rauch geschwärzt und in dieser Art und Weise "vermummt und maskiert" ("vermomt en in mascarade") nach Rom, Venedig, Amsterdam und anderen Orten geschickt haben; in der Folge wurde nicht nur nach dem Schöpfer des Blattes gefragt, sondern vielmehr Goltzius' Kunst mit den besten Arbeiten Dürers in eine rivalisierende Verbindung gebracht. ${ }^{63}$

Wahrscheinlich angeregt durch das von Matham gestochene Bild schuf ein unbekannter Bildschnitzer ein Relief, das sich heute in Wien befindet (Abb. 9). ${ }^{64}$ Dürers Monogramm und die Jahreszahl zeigt das Relief wie Brueghels Tafelgemälde auf der den Betrachtern zugewandten Seite des Felsbrockens. Der unbekannte Schnitzer übertrug Mathams virtuosen Kupferstich in ein skulpturales Medium, das mit primär religiösen Werten verbunden wurde. Anstelle des Täfelchens mit Dürers Monogramm findet sich im Relief eine gefaltete Tafel, die auffällig leer ist, die aber aller Wahrscheinlichkeit nach für den Kreuzestitel bestimmt war. Die Überlagerung von Kreuzestitel und Künstlermonogramm demonstriert vielleicht am deutlichsten die Schwierigkeit, die Autorschaft eines Golgatha-Bildes zu bestimmen, das, wie Golgatha selbst, performativ immer wieder neu hergestellt wird.

\section{Coda}

Um 1600 gehörte Dürers Zeichnung des Großen Kalvarienbergs zu den am meisten verehrten Bildern der Meditation über die Passion. Im Laufe des 16. und frühen 17. Jahrhundert wurde sie intensiv kopiert, zitiert sowie in anderen Medien

62 Wandrey 2017, S. 35-41; Michels 2017, S. 242-248 (Petra Wandrey, mit Angabe der neueren Literatur).

63 Van Mander 1604, Fol. 284v.

64 Beck / Bol 1982, S. 164, Kat. 97 (Bernhard Decker). und Kontexten präsentiert. Die sehr unterschiedlichen Reaktionen auf Dürers Großen Kalvarienberg in den europäischen Kunstzentren der Zeit - Antwerpen, Prag, Haarlem, Florenz - verweisen vor allem auch auf eine intensive Beschäftigung mit altem Wissen über Kunst und künstlerische Materialien am Beispiel des zentralen christlichen Meditationsbildes überhaupt. Dabei reicht es nicht aus, das Kopieren und Nachahmen von Dürers Großem Kalvarienberg ausschließlich im Sinne des traditionellen Verständnisses von Imitation als Emulation zu beurteilen. Dürers Zeichnung fungierte vielmehr auch im historischen Sinne als "principael": Vergleichbar mit einem Früchte tragenden Saatgut oder Zinsen abwerfenden Kapital motivierte Dürers Zeichnung neue Bilder, die ihr bis zu einem gewissen Grad ähnlich waren, sich aber dennoch - im Medium, Material und artificium des ausführenden Künstlers - von ihr unterschieden; die (aufgrund dieser Ähnlichkeit) ihren künstlerischen Ursprung offenbarten, jedoch auch neue Verbindungen und Kontexte eingingen, sodass sie (am Ende) wiederum ihre eigenen Geschichten der Übertragung und Rezeption besaßen. Jan Brueghel der Ältere und Jacob Matham machten auf die andauernde Entstehungsgeschichte durch Monogramme, Signaturen und Jahreszahlen aufmerksam. Brueghels gemalte Kopie blieb darüber hinaus bis heute mit Dürers Zeichnung verbunden. Sowohl in Prag als auch in Florenz war das Bilderpaar Teil großangelegter Sammlungen, die neben lokalen Kunstobjekten auch Artefakte aus allen Teilen der Welt enthielten. Die am Ende des 16. Jahrhunderts errichtete achteckige Tribuna war der zentrale Raum der mediceischen Kunstkammer, an den sich die Waffensammlung, die Sammlungen wissenschaftlicher Instrumente und der Naturobjekte sowie die Werkstätten anschlossen. In die Tribuna gelangten die wertvollsten Sammelgegenstände, d.h. diejenigen Objekte aus allen Zeiten und allen Teilen der Welt, die durch ein hohes Maß an Kunstfertigkeit und Wissen hervorgebracht worden waren. ${ }^{65}$ Als Produkt einer nordeuropäischen Form des chiaroscuro und dessen Aktualisierung durch die Ölmalerei repräsentierte das Bilderpaar jene lokale künstlerische Virtuosität, die in einer zunehmend, global' ausgerichteten Welt Neugier und Faszination erzeugte.

65 Heikamp 1963; Gaeta Bertelà 1997; Turpin 2013. 


\section{Literaturverzeichnis}

\section{Quellen}

Crivelli, Giovanni (1868): Giovanni Brueghel pittor fiammingo o Sue lettere e quadretti esistenti presso I'Ambrosiana. Mailand: Pogliani di E. Besozzi. Erasmus Roterodamus (1528): Dialogus de recta Latini Graecique sermonis pronuntiatione. Basel: Froben.

Kiel, Cornelis (1599): Etymologicum Teutonicae linguae sive Dictionarium Teutonico-latinum. Antwerpen: Moretus.

Lessius, Leonardus (1999 [1605]): Ökonomie und Ethik aus "De iustitia et iure". Faksimile-Ausgabe, Textauswahl von Toon van Houdt. Düsseldorf: Wirtschaft und Finanzen.

Plinius Secundus d. Ä., Caius (1997): Naturkunde. Lateinisch - deutsch. Buch XXXV: Farben, Malerei, Plastik. Hg. und übers. von Roderich König in Zusammenarbeit mit Gerhard Winkler. Darmstadt: Wissenschaftliche Buchgesellschaft.

Quintilianus, Marcus Fabius (1995): Ausbildung des Redners. Zwölf Bücher. Hg. und übers. von Helmut Rahn, 2 Bde. Darmstadt: Wissenschaftliche Buchgesellschaft.

Van Mander, Karel (1604): Het Schilder-Boeck waer in Voor eerst de leerlustighe Iueght den grondt der Edel Vry Schilderconst in Verscheyden deelen Wort Voorghedraghen: Daer nae in dry deelen t'leven der vermaerde doorluchtighe Schilders des ouden, en nieuwen tyds. Eyntlyck d'wtlegghinghe op den Metamorphoseon pub. Ovidij Nasonis. Oock daerbeneffens wtbeeldinghe der figueren. Haarlem: Paschier van Wesbusch.

Von Sandrart, Joachim (1675): Teutsche Academie der Edlen Bau-, Bild- und Mahlerey-Künste. Nürnberg: Johann-Philipp Miltenberger.

\section{Forschungsliteratur}

Baeck, Louis (1999): Die rechtlichen und scholastischen Wurzeln des ökonomischen Denkens von Leonardus Lessius. In: Lessius, Leonardus (1999 [1605]): Ökonomie und Ethik aus „De iustitia et iure". Faksimile-Ausgabe, Textauswahl von Toon van Houdt. Düsseldorf: Wirtschaft und Finanzen, S. 39-78.

Barocchi, Paola / Gaeta Bertelà, Giovanna (Hgg.) (2005): Collezionismo mediceo e storia artistica, Bd. 2. Florenz: Studio per Edizioni Scelte.

Bartrum, Giulia (Hg.) (2002): Albrecht Dürer and His Legacy. The Graphic Work of a Renaissance Artist. Ausstellungskatalog, British Museum. London: British Museum.

Bauer, Rotraud / Haupt, Herbert (1976): Das Kunstkammerinventar Kaiser Rudolfs II., 1607-1611. In: Jahrbuch der kunsthistorischen Sammlungen in Wien, 72, S. 11-191.

Beck, Herbert / Bol, Peter C. (Hgg.) (1982): Dürers Verwandlung in der Skulptur zwischen Renaissance und Barock. Ausstellungskatalog, Liebighaus Museum alter Plastik. Frankfurt a. M.: Liebighaus.

Bedoni, Stefania (1983): Jan Brueghel in Italia e il collezionismo del seicento. Florenz / Mailand: Istituto olandese di storia dell'arte, Università degli studi.

Bodart, Didier (1977): Rubens e la pittura fiamminga del Seicento nelle collezioni pubbliche fiorentine, Ausstellungskatalog, Palazzo Pitti. Florenz: Centro Di.

Bubenik, Andrea (2013): Reframing Dürer: The Appropriation of Art, 1528-1700. Farnham: Ashgate.

Bukovinská, Beket (1986): Die Kunst- und Schatzkammer Rudolfs II.: Der Weg vom Rohmaterial zum Sammlungsobjekt als ein Erkenntnisprozess. In: Liskar, Elisabeth (Hg.): Der Zugang zum Kunstwerk. Schatzkammer, Salon, Ausstellung, "Museum”. Wien: Böhlau, S. 59-63.

Bukovinská, Beket (2003): Bekannte - unbekannte Kunstkammer Rudolfs II. In: Schramm, Helmar / Schwarte, Ludger / Lazardig, Jan (Hgg.): Kunstkammer - Laboratorium - Bühne. Schauplätze des Wissens im 17. Jahrhundert. Berlin / New York: de Gruyter (= Theatrum Scientarum; 1), S. 199-225.

Bukovinská, Beket (2007): Die Kunstkammer Rudolfs II. Umriss der Forschungsgeschichte und Bibliographie. In: Studia Rudolphina, 7, S. 143-167.

Burg, Tobias (2007): Die Signatur. Formen und Funktionen vom Mittelalter bis zum 17. Jahrhundert. Berlin: LIT (= Kunstgeschichte; 80).

Bushart, Magdalena (2018): Intermedialität um 1500. Wechselwirkungen zwischen Druckgraphik und Malerei in der Kunst der Dürerzeit. In: Fajt, Jiří / Jaeger, Susanne (Hgg.): Das Expressive in der Kunst. Albrecht Altdorfer und seine Zeitgenossen. Berlin / München: Deutscher Kunstverlag, S. 43-53.

Dackerman, Susan (Hg.) (2002): Painted Prints. The Revelation of Color in Northern Renaissance \& Baroque Engravings, Etchings \& Woodcuts. Ausstellungskatalog, The Baltimore Museum of Art. University Park, PA: Pennsylvania State University Press.

DaCosta Kaufmann, Thomas (1985): Hermeneutics in the History of Art. Remarks on the Reception of Dürer in the Sixteenth and Early Seventeenth Centuries. In: Chipps Smith, Jeffrey (Hg.): New Perspectives on the Art of Renaissance Nuremberg. Five Essays. Austin, TX: The Archer M. Huntington Art Gallery, S. 22-39.

De Clippel, Karolien / Van der Linden, David (2015): The Genesis of the Netherlandish Flower Piece. Jan Brueghel, Ambrosius Bosschaert and Middelburg. In: Simiolus, 38/1-2, S. 73-86.

Decock, Wim (2009): Lessius and the Breakdown of the Scholastic Paradigm. In: Journal of the History of Economic Thought, 31/1, S. 57-78.

De Marchi, Neil / Van Miegroet, Hans J. (1996): Pricing Invention: "Originals", "Copies”, and their Relative Value in Seventeenth Century Netherlandish Art Markets. In: Ginsburgh, Victor A. / Menger, Pierre-Michel (Hgg.): Economics of the Arts. Selected Essays. Amsterdam: Elsevier, S. 27-70.

Demonts, Louis (1937-1938): Inventaire général des dessins des Écoles du Nord: Écoles allemande et 
suisse, 2 Bde. Paris: Musées Nationaux, Palais du Louvre.

Dubois, Hélène (2017): Michiel Coxcie's Copy as a Formal Reference of the Material Condition of the Ghent Altarpiece. In: Kemperdick, Stephan / Rößler, Johannes / Heyder, Joris Corin (Hgg.): Der Genter Altar. Reproduktionen, Deutungen, Forschungskontroversen. Petersberg: Imhof, S. 92-107.

Duverger, Erik (Hg.) (1984): Antwerpse kunstinventarissen uit de zeventiende eeuw, Bd. 1. Brüssel: Paleis der Academiën.

Ertz, Klaus / Nitze-Ertz, Christa (2008-2010): Jan Brueghel der Ältere (1568-1625). Kritischer Katalog der Gemälde, 4 Bde. Lingen: Luca.

Fehrenbach, Frank (2005): Kohäsion und Transgression. Zur Dialektik lebendiger Bilder. In: Pfisterer, Ulrich / Zimmermann, Anja (Hgg.): Animationen / Transgressionen. Das Kunstwerk als Lebewesen. Berlin: Akademie, S. 1-40.

Filedt Kok, Jan Piet (1996): Over de Calvarieberg. Albrecht Dürer in Leiden, omstreeks 1520. In: Bulletin van het Rijksmuseum, 44/2, S. 335-359.

Fučíková, Eliška (1986): Die Kunstkammer und Galerie Kaiser Rudolfs II. als eine Studiensammlung. In: Liskar, Elisabeth (Hg.): Der Zugang zum Kunstwerk. Schatzkammer, Salon, Ausstellung, "Museum". Wien: Böhlau (= Akten des Internationalen Kongresses für Kunstgeschichte; 4), S. 53-58.

Gaeta Bertelà, Giovanna (1997): La Tribuna di Ferdinando I de' Medici. Inventari 1589-1631. Modena: Panini (= Collezionismo e storia dell'arte; 5).

Göttler, Christine (1990): Die Disziplinierung des Heiligenbildes durch altgläubige Theologie nach der Reformation. Ein Beitrag zur Theorie des Sakralbildes im Übergang vom Mittelalter zur Frühen Neuzeit. In: Scribner, Robert W. / Warnke, Martin (Hgg.): Bilder und Bildersturm im Spätmittelalter und in der frühen Neuzeit. Wiesbaden: Harrassowitz (= Wolfenbütteler Forschungen; 46).

Göttler, Christine / Meganck, Tine (2015): Sites of Art, Nature and the Antique in the Spanish Netherlands. In: Dupré, Sven / De Munck, Bert / Thomas, Werner et al. (Hgg.): Embattled Territory. The Circulation of Knowledge in the Spanish Netherlands. Gent: Academia Press, S. 333-370.

Göttler, Christine (2017): Vulcan's Forge. The Sphere of Art in Early Modern Antwerp. In: Dupré, Sven / Göttler, Christine (Hgg.): Knowledge and Discernment in the Early Modern Arts. New York: Routledge, S. 52-87.

Heikamp, Detlef (1963): Zur Geschichte der UffizienTribuna und der Kunstschränke in Florenz und Deutschland. In: Zeitschrift für Kunstgeschichte, 26, S. $193-268$

Hess, Daniel / Eser, Thomas (Hgg.) (2012): Der frühe Dürer. Ausstellungskatalog, Germanisches Nationalmuseum. Nürnberg: Germanisches Nationalmuseum.

Hirakawa, Kayo (2009): The Pictorialization of Dürer's Drawings in Northern Europe in the Sixteenth and Seventeenth Centuries (= European University
Studies, series XXVIII: History of Art; 434). Bern / New York: Lang.

Honig, Elizabeth Alice (2016): Jan Brueghel and the Senses of Scale. University Park, PA: The Pennsylvania State University Press.

Keller, Katrin (2012): Erzherzogin Maria von Innerösterreich (1551-1608) zwischen Habsburg und Wittelsbach. Wien: Böhlau.

Kemperdick, Stephan / Rößler, Johannes (Hgg.) (2014): Der Genter Altar der Brüder Van Eyck. Geschichte und Würdigung. Ausstellungskatalog, Gemäldegalerie, Staatliche Museen zu Berlin. Petersberg: Imhof.

Kemperdick, Stephan (2014): Die Geschichte des Genter Altars. In: Kemperdick, Stephan / Rößler, Johannes (Hgg.): Der Genter Altar der Brüder Van Eyck. Geschichte und Würdigung. Ausstellungskatalog, Gemäldegalerie, Staatliche Museen zu Berlin. Petersberg: Imhof, S. 8-69.

Kemperdick, Stephan / Rößler, Johannes / Heyder, Joris Corin (Hgg.) (2017): Der Genter Altar: Reproduktionen, Deutungen, Forschungskontroversen. Petersberg: Imhof.

Koerner, Joseph Leo (2016): Bosch \& Bruegel. From Enemy Painting to Everday Use. Princeton / Oxford: Princeton University Press.

Lossky, Boris (1959): Peintures inspirées du Grand Calvaire d'Albert Dürer. In: La Revue des Arts, 9/4-5, S. $158-162$.

Marrow, James H. (1979): Passion Iconography in Northern European Art of the Late Middle Ages and Early Renaissance. A Study of the Transformation of Sacred Metaphor into Descriptive Narrative. Kortrijk: Van Ghemmert.

Mensger, Ariane (2010): Die exakte Kopie. Oder: Die Geburt des Künstlers im Zeitalter seiner Reproduzierbarkeit. In: Chapman, H. Perry / Woodall, Joanna (Hgg.): Envisioning the Artist in the Early Modern Netherlands. Zwolle: Waanders (= Netherlands Yearbook for History of Art; 59), S. 194-221.

Michels, Norbert (Hg.) (2017): Hendrick Goltzius (1558-1617): Mythos, Macht und Menschlichkeit. Aus den Dessauer Beständen. Ausstellungskatalog, Anhaltische Gemäldegalerie Dessau. Petersberg: Imhof.

Nagel, Alexander / Wood, Christopher S. (2010): Anachronic Renaissance. New York: Zone Books.

Neumeister, Mirjam (Hg.) (2013): Brueghel. Gemälde von Jan Brueghel d. Ä., Bayerische Staatsgemäldesammlungen, München. München: Hirmer.

Neumeister, Mirjam / Ortner, Eva / Schmidt, Jan (2013): Der Blick auf das Detail - Zur Maltechnik Jan Brueghels d. Ä. In: Neumeister, Mirjam (Hg.): Brueghel. Gemälde von Jan Brueghel d. Ä., Bayerische Staatsgemäldesammlungen, München. München: Hirmer, S. 91-107.

Panofsky, Erwin (1951): „Nebulae in Pariete”. Notes on Erasmus' Eulogy on Dürer. In: Journal of the Warburg and Courtauld Institutes, 14, S. 34-41.

Putzger, Antonia (2018): Distinktion von Stilen und Stil als Distinktion. Zur retrospektiven Kategorisierung 
der mañera flamenca und deren Aneignung durch die spanischen Habsburger. In: Blunk, Julian / Michalsky, Tanja (Hgg.): Stil als (geistiges) Eigentum. München: Hirmer, S. 105-123.

Putzger, Antonia (2019): Rückgriff oder Simulation? Zu frühneuzeitlichen Kopierverfahren am Beispiel Michiel Coxcies. In: Bushart, Magadalena / Haug, Henrike / Stallschus, Stefanie (Hgg.): Unzeitgemäße Techniken. Historische Narrative künstlerischer Verfahren. Wien / Köln / Weimar: Böhlau (= Interdependenzen; 4), S. 73-95.

Reitz, Evelyn (2015): Discordia concors. Kulturelle Differenzerfahrung und ästhetische Einheitsbildung in der Prager Kunst um 1600. Berlin / Boston: de Gruyter.

Roth, Elisabeth (1958): Der volkreiche Kalvarienberg in Literatur und Kunst des Spätmittelalters. Berlin: Schmidt.

Silver, Larry (2008): Translating Dürer into Dutch. In: Chapuis, Julien (Hg.): Invention. Northern Renaissance Studies in Honor of Molly Faries. Turnhout: Brepols, S. 209-223.

Strauss, Walter L. (1974): The Complete Drawings of Albrecht Dürer, 6 Bde. New York: Abaris Books.

Suckale, Robert (2009). Die Erneuerung der Malkunst vor Dürer, 2 Bde. Petersberg: Imhof.

Suykerbuyk, Ruben (2013): Coxcie's Copies of Old Masters. An Addition and an Analysis. In: Simiolus. Netherlands Quarterly for the History of Art, 37/1, S. 5-24.

Suykerbuyk, Ruben (2017): A Royal Reproduction. Michiel Coxcie's Copy of the Ghent Altarpiece (1557-1558), its Documentary Evidence and Early Reception. In: Kemperdick, Stephan / Rößler, Johannes / Heyder, Joris Corin (Hgg.): Der Genter Altar. Reproduktionen, Deutungen, Forschungskontroversen. Petersberg: Imhof, S. 70-83.

Turpin, Adriana (2013): The Display of Exotica in the Uffizi Tribuna. In: Bracken, Susan / Gáldy, Andrea / Turpin, Adriana (Hgg.): Collecting East and West. Newcastle upon Tyne: Cambridge Scholars, S. 83-118.

Van Houdt, Toon (1999): Zusammenfassung der Gedanken über Ökonomie und Ethik aus De iustitia et iure von Leonardus Lessius. In: Schefold, Bertram (Hg.): Vademecum zu einem Klassiker der spätscholastischen Wirtschaftsanalyse. Düsseldorf: Wirtschaft und Finanzen, S. 103-142.
Van Os, Henk (1996): Mediteren op Golgotha: „O devote siele slaet dyn gemerck hierop dinen bruidegom". In: Bulletin van het Rijksmuseum, 44, S. 361-380.

Van Os, Henk (2001): Another Meditation on Golgatha. In: Bergdolt, Klaus / Bonsanti, Giorgio (Hgg.): Opere e giorni: Studi su mille anni di arte europea. Venedig: Marsilio, S. 479-484.

Von Rosen, Valeska (2000): Die Enargeia des Gemäldes. Zu einem vergessenen Inhalt des Ut-picturapoesis und seiner Relevanz für das cinquecenteske Bildkonzept. In: Marburger Jahrbuch für Kunstwissenschaft, 27, S. 171-208.

Wandrey, Petra (2017): Schönheit bändigt allen Zorn die Meisterstiche von Hendrick Goltzius. In: Michels, Norbert (Hg.): Hendrick Goltzius (1558-1617): Mythos, Macht und Menschlichkeit. Aus den Dessauer Beständen. Ausstellungskatalog, Anhaltische Gemäldegalerie Dessau. Petersberg: Imhof, S. 34-41.

White, Christopher (1971): Dürer. The Artist and his Drawings. London: Phaidon.

Widerkehr, Léna (2007-2008): Jacob Matham, 3 Bde. Oudekerk aan den Ijssel: Sound \& Vision (= The New Hollstein Dutch \& Flemish Etchings, Engravings and Woodcuts; 50-52).

Windows, Peter (2012): New Identifications in the Drawings Collection of Gabriele Vendramin. In: Master Drawings, 50/1, S. 33-48.

Winkler, Friedrich (1929): Dürerstudien. In: Jahrbuch der Preußischen Kunstsammlungen, 50, S. 123-166.

Winkler, Friedrich (1936-1939): Die Zeichnungen Albrecht Dürers, 4 Bde. Potsdam: Stechnote. 